\title{
A $q$-ANALOGUE OF CONVOLUTION ON THE LINE*
}

\author{
G. CARNOVALE ${ }^{\dagger}$ AND T. H. KOORNWINDER ${ }^{\ddagger}$
}

\begin{abstract}
In this paper we study a $q$-analogue of the convolution product on the line in detail. A convolution product on the braided line was defined algebraically by Kempf and Majid. We adapt their definition in order to give an analytic definition for the $q$-convolution and we study convergence extensively. Since the braided line is commutative as an algebra, all results can be viewed both as results in classical $q$-analysis and in braided algebra. We define various classes of functions on which the convolution is well-defined and we show that they are algebras under the defined product. One particularly nice family of algebras, a decreasing chain depending on a parameter running through $(0,1]$, turns out to have $1 / 2$ as the critical parameter value above which the algebras are commutative. Morerover, the commutative algebras in this family are precisely the algebras in which each function is determined by its $q$-moments.

We also treat the relationship between $q$-convolution and $q$-Fourier transform. Finally, in the Appendix, we show an equivalence between the existence of an analytic continuation of a function defined on a $q$-lattice, and the behaviour of its $q$-derivatives.
\end{abstract}

1. Introduction. The classical Fourier transform $\mathcal{F}$ and the classical convolution product are closely tied to each other by the homomorphism property $\mathcal{F}(f * g)=$ $\mathcal{F}(f) \mathcal{F}(g)$, while both operations have a conceptual interpretation on the real line $\mathbf{R}$ considered as locally compact abelian group. Classical convolution is of great importance, both in theory and in applications. Whenever one has an interesting generalization of the classical Fourier transform (like the $q$-Fourier transform in the present paper), it is therefore natural to consider an analogue of classical convolution which is related to the generalized Fourier transform in a similar way as classical convolution is related to classical Fourier transform. The present paper and its sequel will consider convolution related to a $q$-Fourier transform involving the $q$-exponential function $E_{q}$ as a kernel. This transform was analytically introduced by the second author in [11], Section 8, and it was earlier considered algebraically by Kempf and Majid [7], where it occurs as the special case for the braided line of their general theory of Fourier transform on "braided covector algebras". The braided line, a deformation as a braided group of the algebra of functions on $\mathbf{R}$, is the simplest non-trivial example of a braided covector algebra. It was first introduced in a rudimental way by the second author in Section 6.8 of [10] and in full detail by S. Majid, see [13], [14]. The Fourier transform on a braided covector algebra of type $A_{n}$ (a braided analogue of function space on $\mathbf{R}^{n}$ ) was studied in more detail by the first author in [3].

In [7] Kempf and Majid also defined convolution for those braided covector algebras which have an integral which is bosonic and invariant under translation. A slight adaptation of their definition in case of the braided line (where the integral is not bosonic) is the starting point of our analytic definition of $q$-convolution $f *_{\gamma} g$ on $\mathbf{R}$ given below (here $\gamma$ denotes the choice of a $q$-lattice). Two other motivations for this definition can be given: the formal limit for $q \rightarrow 1$ yields classical convolution, and $\mathcal{F}_{\gamma}\left(f *_{\gamma} g\right)=\left(\mathcal{F}_{\gamma} f\right)\left(\mathcal{F}_{\gamma} g\right)$ holds if we take for $\mathcal{F}_{\gamma}$ the $q$-Fourier transform of [11]

${ }^{*}$ Received Sept. 16, 1999; revised August 8, 2000.

†Dipartimento di Matematica Pura ed Applicata, Università degli Studi di Padova, via Belzoni 7, 35131 Padova, Italy (carnoval@math.unipd.it). Research for this paper was started while the first author worked at the University of Utrecht, supported by NWO, project number 610-06-100. The paper was completed while she worked at the University of Cergy-Pontoise, supported by the University of Trieste.

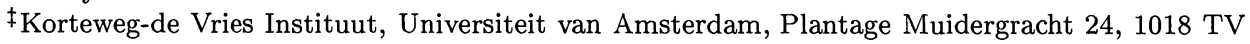
Amsterdam, The Netherlands (thk@science.uva.nl). 
involving $E_{q}$ as a kernel.

The aim of the present paper is to find suitable function spaces for $f$ and $g$ such that their $q$-convolution $f *_{\gamma} g$ is well-defined, and to find function classes on which associativity and commutativity hold. Let us introduce some notation for explaining this.

Throughout this paper $q$ is fixed such that $0<q<1$. The $q$-derivative of a function $f$ is given by $(\partial f)(x):=\frac{f(x)-f(q x)}{(1-q) x}$, and its $q$-shift by $(Q f)(x):=f(q x)$. For $\gamma>0$ we have the $q$-lattice $L(\gamma):=\left\{ \pm q^{k} \gamma \mid k \in \mathbf{Z}\right\}$. For a function $f$ on $L(\gamma)$ the (unbounded) $q$-integral over $L(\gamma)$ is denoted and defined by

$$
\int_{\gamma} f=\int_{-\gamma \cdot \infty}^{\gamma \cdot \infty} f(t) d_{q} t:=(1-q) \sum_{k=-\infty}^{\infty} \sum_{\epsilon= \pm 1} q^{k} \gamma f\left(\epsilon q^{k} \gamma\right)
$$

provided the summation absolutely converges.

Fix $\gamma>0$. Let $\mathcal{I}_{\gamma}$ denote the space of absolutely $q$-integrable functions on $L(\gamma)$, and let $\mathcal{I}_{\gamma}^{\infty}$ denote the subspace of functions $f \in \mathcal{I}_{\gamma}$ such that $x \mapsto f(x) x^{e}$ is in $\mathcal{I}_{\gamma}$ for every $e \in \mathbf{Z}_{\geq 0}$. For $f \in \mathcal{I}_{\gamma}^{\infty}$ define the moments, respectively strict moments of $f$ by:

$$
\mu_{e, \gamma}(f):=q^{\frac{e^{2}+e}{2}} \int_{-\gamma \cdot \infty}^{\gamma \cdot \infty} f(x) x^{e} d_{q} x, \quad \nu_{e, \gamma}(f):=q^{\frac{e^{2}+e}{2}} \int_{-\gamma \cdot \infty}^{\gamma \cdot \infty}\left|f(x) x^{e}\right| d_{q} x
$$

The factor $q^{\frac{e^{2}+e}{2}}$ is a normalization factor by means of which most statements and formulas can be expressed in a simplified form (see Section 4).

DEFINITION 1.1. Let $f \in \mathcal{I}_{\gamma}^{\infty}$ and let $g$ be a function defined on some subset of C. Then the $q$-convolution product $f *_{\gamma} g$ is the function given by

$$
\left(f *_{\gamma} g\right)(x):=\sum_{e=0}^{\infty} \frac{(-1)^{e} \mu_{e, \gamma}(f)}{[e]_{q} !}\left(\partial^{e} g\right)(x)
$$

for $x \in \mathbf{C}$ such that the $q$-derivatives $\left(\partial^{e} g\right)(x)$ are well-defined for all $e \in \mathbf{Z}_{\geq 0}$ and the sum on the right converges absolutely, and with notation (1.2) being used.

By the asymmetric form of our definition of $f *_{\gamma} g$, the initial choices of function classes for $f$ and $g$ are quite different: for $f$ the moments $\mu_{e, \gamma}(f)$ should behave as $O\left(q^{\frac{\alpha e^{2}}{2}} b^{e}\right)$ for $e \rightarrow \infty$ with $\alpha, b>0$ (we call this of left type $\alpha$ ), while for $g$ the $q$-derivatives $\left(\partial^{k} g\right)(x)$ at $x$ should behave as $O\left(R^{k}\right)$ for $k \rightarrow \infty$ with $R>0$. We also need functions on $L(\gamma)$ which extend to analytic functions, on a disk centered at 0 , or on a strip around $\mathbf{R}$. Some equivalent characterizations of function spaces derived in this paper may have independent interest (see for instance the Appendix).

Commutativity of the convolution product is the hardest and most interesting issue of this paper. Both the homomorphism property and the interpretation on the (commutative) braided line suggest commutativity, but we find significant counterexamples. An explanation is that the $q$-Fourier transform of $f$ only depends on the moments of $\mu_{e, \gamma}(f)$ and that $\mu_{e, \gamma}\left(f *_{\gamma} g\right)$ is symmetric in $f$ and $g$, but that $f$ in certain function classes is not completely determined by its moments. For getting commutativity we need functions $f$ (and $g$ ) in the convolution product for which the strict moments $\nu_{e, \gamma}(f)$ behave as $O\left(q^{\frac{\alpha e^{2}}{2}} b^{e}\right)$ for $e \rightarrow \infty$ with $\alpha>1 / 2, b>0$ (we call this of strict left type $\alpha>1 / 2$ ), and which are holomorphic on a strip around $\mathbf{R}$. It 
turns out that the $q$-Gaussian $x \mapsto e_{q^{2}}\left(-x^{2}\right)$, which has has strict left type $1 / 2$, does not commute with many entire functions of strict left type $>1 / 2$.

Part of the results of this paper occurred in the recent dissertation [4] by the first author. She discusses some further aspects of $q$-convolution in the subsequent paper $[5]$.

ACKNowledgement. We thank the referee for his careful reading of the manuscript and his constructive remarks.

FURTHER NOTATIONS. We denote as usual:

$$
\begin{gathered}
(a ; q)_{k}:=\prod_{j=0}^{k-1}\left(1-a q^{j}\right), \quad(a ; q)_{\infty}:=\lim _{k \rightarrow \infty}(a ; q)_{k}, \\
{[k]_{q}:=\frac{1-q^{k}}{1-q}, \quad[k]_{q} !:=\frac{(q ; q)_{k}}{(1-q)^{k}}, \quad\left[\begin{array}{l}
k \\
j
\end{array}\right]_{q}:=\frac{[k]_{q} !}{[j]_{q} ![k-j]_{q} !}=\frac{(q ; q)_{k}}{(q ; q)_{j}(q ; q)_{k-j}} .}
\end{gathered}
$$

A function $f: x \mapsto f(x)$ may also be denoted as $f(X)$. This will be useful for functions like $f X^{e}: x \mapsto f(x) x^{e}$ and $e_{q^{2}}\left(-X^{2}\right): x \mapsto e_{q^{2}}\left(-x^{2}\right)$.

For $q$-hypergeometric series the notation of Gasper \& Rahman [6] will be followed.

The (bounded) $q$-integral of a function $f$ on $\left\{ \pm \gamma q^{k} \mid k \in \mathbf{Z}_{\geq 0}\right\}$ is denoted and defined by

$$
\int_{-\gamma}^{\gamma} f(t) d_{q} t=(1-q) \sum_{k=0}^{\infty} \sum_{\epsilon= \pm 1} q^{k} \gamma f\left(\epsilon q^{k} \gamma\right)
$$

provided the summation absolutely converges.

2. Motivation of the definition of $q$-convolution. We will give three different motivations for our Definition 1.1 of $q$-convolution in the following three remarks.

REMARK 2.1. The braided line (see [14]) is a braided Hopf algebra $\mathcal{A}$ which, as an algebra, is equal to the (commutative) algebra $\mathbf{C}[[x]]$ of formal power series in $x$, and which has braiding $\Phi\left(x^{k} \otimes x^{l}\right):=q^{k l} x^{l} \otimes x^{k}$, comultiplication $\Delta\left(x^{k}\right):=$ $\sum_{j=0}^{k}\left[\begin{array}{l}k \\ j\end{array}\right]_{q} x^{k-j} \otimes x^{j}$, counit $\varepsilon\left(x^{k}\right):=\delta_{k, 0}$ and braided antipode $S\left(x^{k}\right):=(-1)^{k} q^{\frac{k^{2}-k}{2}} x^{k}$. Then the $q$-analogue of Taylor's formula is given by

$$
\Delta(f(x))=\sum_{j=0}^{\infty} \frac{x^{j}}{[j]_{q} !} \otimes \partial^{j}(f(x)),
$$

where $\partial$ denotes the $q$-derivative.

The original formal definition of a convolution on any braided covector algebra $A$ with a bosonic integral $\int: A \rightarrow \mathbf{C}$ invariant under translation, was given by Kempf \& Majid [7] as follows:

$$
(f * g)(x):=\left(\int \otimes \mathrm{id}\right)(m \otimes \mathrm{id})(\mathrm{id} \otimes S \otimes \mathrm{id})(\mathrm{id} \otimes \Delta)(f \otimes g)(x) .
$$

In the case of the braided line we take for $\int$ the integral defined by (1.1). This integral is invariant under translation (see [7], and [11] and [3] for an analytic proof), but it is not bosonic. Associativity will fail for the convolution defined by (2.2). Therefore, we slightly modify (2.2) into

$$
\left(f *_{\gamma} g\right)(x):=\left(\int \otimes \mathrm{id}\right)(m \otimes \mathrm{id})(\mathrm{id} \otimes Q \otimes \mathrm{id})(\mathrm{id} \otimes S \otimes \mathrm{id})(\mathrm{id} \otimes \Delta)(f \otimes g)(x) .
$$


When we substitute the $q$-Taylor formula (2.1) into (2.3) then we formally get formula (1.3) (with (1.2) substituted), i.e., our original definition of $q$-convolution.

We may interpret $f *_{\gamma} g$ formally as the action of a pseudo- $q$-differential operator $\mathcal{D} f(\partial)$ on $g(x)$, where $\mathcal{D} f(\partial):=\mathcal{F}_{S}^{\prime}(\gamma)(f)(i(1-q) \partial)$. Here $\mathcal{F}_{S}^{\prime}(\gamma)(f):=$ $\sum_{e=0}^{\infty} \frac{(-1)^{e} \mu_{e, \gamma}(f)}{[e]_{q} !} \otimes \partial^{e}$ is the weak braided Fourier transform considered in [3] (with $q^{2}$ instead of $q$ ).

REMARK 2.2. The formal limit for $q \uparrow 1$ of formula (1.3) is the classical convolution product:

$$
\begin{gathered}
\sum_{e=0}^{\infty}\left(\int_{-\infty}^{\infty} d t f(t) \frac{(-1)^{e} t^{e}}{e !}\right) g^{(e)}(x) \stackrel{\text { formally }}{=} \int_{-\infty}^{\infty} d t\left(f(t)\left(\sum_{e=0}^{\infty} \frac{(-1)^{e} t^{e}}{e !} g^{(e)}(x)\right)\right) \\
\stackrel{\text { formally }}{=} \int_{-\infty}^{\infty} d t f(t) g(x-t)=(f * g)(x) .
\end{gathered}
$$

REMARK 2.3. Recall the two $q$-exponentials (see [6]):

$$
e_{q}(x):=\sum_{k=0}^{\infty} \frac{x^{k}}{(q ; q)_{k}}=\frac{1}{(x ; q)_{\infty}}, \quad E_{q}(x):=\sum_{k=0}^{\infty} \frac{q^{\frac{k(k-1)}{2}} x^{k}}{(q ; q)_{k}}=(-x ; q)_{\infty}
$$

where $|x|<1$ in the infinite sum defining $e_{q}(x)$. In [11] a $q$-Fourier transform pair was presented as follows:

$$
\psi(x)=\frac{1}{c_{q}(\gamma) b_{q}} \int_{-1}^{1} e_{q}(-i x y) \phi(y) d_{q} y, \quad \phi(y)=\int_{-\gamma . \infty}^{\gamma \cdot \infty} E_{q}(i q x y) \psi(x) d_{q} x
$$

where $b_{q}$ and $c_{q}(\gamma)$ are given by

$$
\begin{aligned}
b_{q} & :=\int_{-1}^{1} E_{q^{2}}\left(-q^{2} x^{2}\right) d_{q} x=(1-q)(q,-q,-1 ; q)_{\infty} \\
c_{q}(\gamma) & :=\int_{-\gamma . \infty}^{\gamma \cdot \infty} e_{q^{2}}\left(-x^{2}\right) d_{q} x=\frac{2(1-q)\left(q^{2},-q \gamma^{2},-q \gamma^{-2} ; q^{2}\right)_{\infty} \gamma}{\left(-\gamma^{2},-q^{2} / \gamma^{2}, q ; q^{2}\right)_{\infty}} .
\end{aligned}
$$

Write the second transform in (2.5) as $\phi=\mathcal{F}_{\gamma} \psi$. Then an immediate formal computation shows that

$$
\left(\mathcal{F}_{\gamma}\left(f *_{\gamma} g\right)\right)(x)=\left(\mathcal{F}_{\gamma} f\right)(x)\left(\mathcal{F}_{\gamma} g\right)(x)
$$

The transform $\mathcal{F}_{\gamma}$ is essentially the weak braided Fourier transform $\mathcal{F}_{S}^{\prime}(\gamma)$ (see Remark 2.1 and reference [3]). Equation (2.8) will be rigorously proved for suitable $f$ and $g$ in Section 7.

For later use we recall the formulas (9.8), (9.14) in [11] (for $n \in \mathbf{Z}_{\geq 0}$ ):

$$
\begin{aligned}
& \int_{-\gamma \cdot \infty}^{\gamma \cdot \infty} x^{n} e_{q^{2}}\left(-x^{2}\right) d_{q} x=c_{q}(\gamma) q^{-k^{2}}\left(q ; q^{2}\right)_{k} \quad \text { if } n=2 k \quad(=0 \text { otherwise }) \\
& \int_{-1 \cdot \infty}^{1 \cdot \infty} x^{n} E_{q^{2}}\left(-x^{2}\right) d_{q} x=b_{q} q^{2 k+1}\left(q ; q^{2}\right)_{k} \quad \text { if } n=2 k \quad(=0 \text { otherwise })
\end{aligned}
$$


3. Good function spaces for $q$-convolution. In general, it is not true that $\left(f *_{\gamma} g\right)(x)$ can be expanded as a (possibly formal) power series if $g(x)$ has a convergent power series expansion. The reason is that the coefficients of an expansion in powers of $x$ of $\left(f *_{\gamma} g\right)(x)$ will be in general infinite series themselves. We want to find conditions on $f$ and $g$ so that, with the given definition, $\left(f *_{\gamma} g\right)(x)$ makes sense on some subset of the complex plane. One should give conditions on the growth of the moments $\left|\mu_{e, \gamma}(f)\right|$ of $f$ and of the power series coefficients of $g$. For this purpose we will now introduce the class of functions of left type $\alpha$. For convenience, we also give here some variants of this definition and corresponding notation which will be needed later in the paper. Recall the definitions of the function spaces $\mathcal{I}_{\gamma}$ and $\mathcal{I}_{\gamma}^{\infty}$, given in the Introduction.

\section{DEFINITION 3.1.}

(a) For $\alpha>0$ the space $\mathcal{I}_{\gamma, \alpha}^{\omega}$ of functions of left type $\alpha$ on $L(\gamma)$ consists of all $f \in \mathcal{I}_{\gamma}^{\infty}$ such that, for some $b>0,\left|\mu_{e, \gamma}(f)\right|=O\left(q^{\frac{\alpha e^{2}}{2}} b^{e}\right)$ as $e \rightarrow \infty$.

The space $\mathcal{I}_{\gamma}^{\omega}$ consists of all functions of some left type $\alpha>0$ on $L(\gamma)$.

(b) For $\alpha>0$ the space $\mathcal{I}_{\gamma, \alpha}^{\mathrm{s} \omega}$ of functions of strict left type $\alpha$ on $L(\gamma)$ consists of all $f \in \mathcal{I}_{\gamma}^{\infty}$ such that, for some $b>0, \nu_{e, \gamma}(f)=O\left(q^{\frac{\alpha e^{2}}{2}} b^{e}\right)$ as $e \rightarrow \infty$.

The space $\mathcal{I}_{\gamma}^{\mathrm{s} \omega}$ consists of all functions of some strict left type $\alpha>0$ on $L(\gamma)$.

(c) For $a>0$ the space $\mathcal{H}_{a}^{\mathrm{D}}$ consists of all functions which are holomorphic on the disk $\{z \in \mathbf{C}|| z \mid<a\}$. By $\mathcal{H}^{\mathrm{D}}$ we denote the space of all functions which are holomorphic on some disk centered at 0 .

(d) For $a>0$ the space $\mathcal{H}_{a}^{\mathrm{S}}$ consists of all functions which are holomorphic on the strip $\{z \in \mathbf{C}|| \operatorname{Im}(z) \mid<a\}$. By $\mathcal{H}^{\mathrm{S}}$ we denote the space of all functions which are holomorphic on some strip around $\mathbf{R}$.

The "intersection" of a $\mathcal{H}$-space defined in (c) or (d) with one of the $\mathcal{I}$-spaces (i.e. $\mathcal{I}_{\gamma}, \mathcal{I}_{\gamma}^{\infty}$ or a space defined in (a) or (b)) will be denoted by putting the two symbols $\mathcal{H}$ and $\mathcal{I}$ behind each other. Here we mean intersection in a special sense. For instance, $\mathcal{H}^{\mathrm{D}} \mathcal{I}_{\gamma}^{\infty}$ will denote the space of the functions on $L(\gamma)$ belonging to $\mathcal{I}_{\gamma}^{\infty}$ which coincide within some disk centered at 0 with the restriction of a (necessarily unique) holomorphic function on that disk. Note that, conversely, a function $f \in \mathcal{H}^{\mathrm{D}} \mathcal{I}_{\gamma}^{\infty}$ is in general not uniquely determined by the function in $\mathcal{H}^{\mathrm{D}}$ with which it has a common restriction within some disk.

We will always assume for the "intersections" just defined that $\gamma$ is less than the parameter $a$ (radius of a disk or half width of a strip) occurring in $\mathcal{H}_{a}^{\mathrm{D}}$ or $\mathcal{H}_{a}^{\mathrm{S}}$. Clearly, this assumption is not restrictive, since $\gamma$ may be replaced by $q^{k} \gamma$ for arbitrary $k \in$ $\mathbf{Z}_{\geq 0}$.

Observe that, if $f \in \mathcal{I}_{\gamma}^{\infty}$ is of left type $\alpha$ (respectively strict left type $\alpha$ ) on $L(\gamma)$ then so is $X^{k} f$ for for each $k \in \mathbf{Z}_{\geq 0}$. Indeed, $\mu_{e, \gamma}\left(f X^{k}\right)=q^{-\frac{k^{2}}{2}-\frac{k}{2}-e k} \mu_{k+e, \gamma}(f)$, and similarly for $\mu$ replaced by $\nu$.

EXAMPLE 3.2.

(a) By (2.9) the $q$-Gaussian $e_{q^{2}}\left(-X^{2}\right)$ belongs to $\mathcal{I}_{\gamma}^{\infty}$ for each $\gamma>0$ and

$$
\mu_{2 k, \gamma}\left(e_{q^{2}}\left(-X^{2}\right)\right)=c_{q}(\gamma)\left(q ; q^{2}\right)_{k} q^{k^{2}+k}, \quad \mu_{2 k+1, \gamma}\left(e_{q^{2}}\left(-X^{2}\right)\right)=0 .
$$

Hence $e_{q^{2}}\left(-X^{2}\right)$ is of left type $1 / 2$ on $L(\gamma)$. It is also of strict left type $1 / 2$ on $L(\gamma)$. Indeed, if $f \in \mathcal{I}_{\gamma}^{\infty}$ is an even and nonnegative function then $\nu_{2 k, \gamma}(f)=\mu_{2 k, \gamma}(f)$ and $\nu_{2 k+1, \gamma}(f) \leq \mu_{2 k+2, \gamma}(f)+\mu_{2 k, \gamma}(f)$. 
(b) By (2.10) the $q$-Gaussian $E_{q^{2}}\left(-X^{2}\right)$ belongs to $\mathcal{I}_{1}^{\infty}$ and

$$
\mu_{2 k, 1}\left(E_{q^{2}}\left(-X^{2}\right)\right)=b_{q}\left(q ; q^{2}\right)_{k} q^{2 k^{2}+k}, \quad \mu_{2 k+1,1}\left(E_{q^{2}}\left(-X^{2}\right)\right)=0 .
$$

Hence $E_{q^{2}}\left(-X^{2}\right)$ is of left type 1 on $L(1)$. It is also of strict left type 1 on $L(1)$ by a similar argument as in (a).

REMARK 3.3. Let $g \in \mathcal{H}_{R}^{\mathrm{D}}$ with $g(x)=\sum_{l} c_{l} x^{l}$ and put $G(r):=\sum_{l}\left|c_{l}\right| r^{l}$ (we will repeatedly use this convention). Note that then also $\partial^{k} g \in \mathcal{H}_{R}^{\mathrm{D}}$ for every $k \in \mathbf{Z}_{\geq 0}$. Since $\left|c_{l}\right| \leq\left(\sum_{p}\left|c_{p}\right| r^{p}\right) r^{-l}$ for every $r<R$ and since $\left(q^{l} ; q^{-1}\right)_{k} \leq 1$ for $l \geq k$ we can also say that, for every $|x|<r<R$,

$$
\left|\left(\partial^{k} g\right)(x)\right| \leq \frac{r}{r-|x|} \frac{1}{r^{k}(1-q)^{k}} G(r) .
$$

It will be shown in the Appendix that condition (3.1) together with equality of left and right $q$-derivatives at 0 is equivalent to analyticity.

LEMMA 3.4. Let $f \in \mathcal{I}_{\gamma, \alpha}^{\omega}$ and $g \in \mathcal{H}_{a}^{\mathrm{D}}$. Then $f *_{\gamma} g \in \mathcal{H}_{a}^{\mathrm{D}}$. If $g$ can be continued analytically on a starlike domain $\Omega$ centered at 0 , then $f *_{\gamma} g$ is well-defined and analytic on $\Omega$.

Proof. We want to show that $\left(f *_{\gamma} g\right)(x)$ has a convergent power series expansion for $|x|<a$. Let $g(x):=\sum_{l} c_{l} x^{l}$. Then direct computation gives

$$
\left(f *_{\gamma} g\right)(x)=\sum_{e=0}^{\infty} \sum_{l=e}^{\infty} \frac{(-1)^{e} \mu_{e, \gamma}(f)}{[e]_{q} !} c_{l} \frac{[l]_{q} !}{[l-e]_{q} !} x^{l-e} .
$$

For every $r<a$ and every $x \in \mathbf{C}$ such that $|x|<r$ one has

$$
\begin{aligned}
\sum_{e=0}^{\infty} \sum_{l=e}^{\infty}\left|\mu_{e, \gamma}(f)\right|\left|c_{l}\right|\left[\begin{array}{l}
l \\
e
\end{array}\right]_{q}|x|^{l-e} & \leq C \sum_{e=0}^{\infty} \sum_{p=0}^{\infty} \frac{q^{\frac{\alpha}{2} e^{2}} b^{e}(q ; q)_{e+p}}{(q ; q)_{p}(q ; q)_{e}} G(r) r^{-p-e}|x|^{p} \\
& \leq G(r) C \sum_{e=0}^{\infty} \frac{q^{\frac{\alpha}{2} e^{2}} r^{-e} b^{e}}{(q ; q)_{e}} \sum_{p=0}^{\infty}\left(\frac{|x|}{r}\right)^{p}<\infty
\end{aligned}
$$

for some $C>0$. Hence, by dominated convergence, we may invert the order of summation in formula (3.2) so that, for $|x|<a$, we have

$$
\left(f *_{\gamma} g\right)(x)=\sum_{p=0}^{\infty}\left(\sum_{e=0}^{\infty}(-1)^{e} \mu_{e, \gamma}(f)\left[\begin{array}{c}
p+e \\
e
\end{array}\right]_{q} c_{p+e}\right) x^{p}=\sum_{p=0}^{\infty} b_{p} x^{p}
$$

where $\left|b_{p}\right| \leq G(r) C r^{-p} \sum_{e=0}^{\infty} \frac{q^{\frac{\alpha}{2} e^{2}}\left(q^{p+1} ; q\right)_{e}}{(q ; q)_{e}} b^{e} r^{-e}<\infty$, while the power series of $\left(f *_{\gamma}\right.$ $g)(x)$ converges absolutely for $|x|<a$.

Let now $g$ be continued analytically on a starlike domain $\Omega$ centered at 0 . It follows by induction that for every starlike compact set $K$ centered at 0 and contained in $\left.\Omega, \mid\left(\partial^{e} g\right)(x)\right) \mid \leq\|g\|_{K} \frac{2^{e}}{|x|^{e}(1-q)^{e}}$ for every nonzero $x \in K$, where $\|\cdot\|_{K}$ denotes the supremum norm. Let $s>0$. Then, for $x \in K$ with $|x| \geq s$,

$$
\left(f *_{\gamma} g\right)(x)=\sum_{e=0}^{\infty} \frac{(-1)^{e} \mu_{e, \gamma}(f)}{[e]_{q} !}\left(\partial^{e} g\right)(x)
$$


with

$$
\left|\frac{(-1)^{e} \mu_{e, \gamma}(f)}{[e]_{q} !}\left(\partial^{e} g\right)(x)\right| \leq C \frac{q^{\frac{\alpha}{2} e^{2}} b^{e}}{[e]_{q} !}\left|\left(\partial^{e} g\right)(x)\right| \leq C \frac{q^{\frac{\alpha}{2} e^{2}}(2 b)^{e}}{s^{e}(q ; q)_{e}} .
$$

Hence, on each set $\{x \in K|| x \mid>s\}$ with $K \subset \Omega$ a starlike compact set centered at 0 and $s>0$, the series expressing $\left(f *_{\gamma} g\right)(x)$ is uniformly convergent, with

$\sum_{e=0}^{E} \frac{(-1)^{e} \mu_{e, \gamma}(f)}{[e]_{q} !}\left(\partial^{e} g\right)(x)$ analytic in $x$ for every $E \in \mathbf{Z}_{\geq 0}$. It follows that $f *_{\gamma} g$ is analytic on each set $\{x \in \Omega|| x \mid>s\}$ with $s>0$. Since we already showed that $f *_{\gamma} g$ is analytic on $\{x \in \mathbf{C}|| x \mid<a\}$, this completes the proof.

Now we consider $g$ in $\mathcal{H}_{a}^{\mathrm{D}} \mathcal{I}_{\gamma}$ or in $\mathcal{H}_{a}^{\mathrm{S}} \mathcal{I}_{\gamma}$ with $a>\gamma$. An example of a function in $\mathcal{H}_{a}^{\mathrm{D}} \mathcal{I}_{\gamma}$ is any function $g$ with finite support contained in $\left\{ \pm q^{-k} \gamma \mid k \in \mathbf{Z}_{\geq 1}\right\}$.

An example of a function in $\mathcal{H}_{a}^{\mathrm{S}} \mathcal{I}_{\gamma}$ is the function $e_{q^{2}}\left(-X^{2}\right)$. It is clearly an element of $\mathcal{H}_{1}^{\mathrm{S}} \mathcal{I}_{\gamma}$ for every $\gamma \in(0,1)$, since $e_{q^{2}}\left(-x^{2}\right)=\sum_{k=0}^{\infty} \frac{(-1)^{k} x^{2 k}}{\left(q^{2} ; q^{2}\right)_{k}}$ for $|x|<1$ and since this function has analytic continuation $\frac{1}{\left(-x^{2} ; q^{2}\right)_{\infty}}$ for $x \neq i q^{-k}\left(k \in \mathbf{Z}_{\geq 0}\right)$.

Lemma 3.5. Let $g \in \mathcal{H}_{a}^{\mathrm{D}} \mathcal{I}_{\gamma}^{\infty}$ for some $a>\gamma$. Then for every $r \in(\gamma, a)$ there is a constant $B>0$ such that

$$
\int_{-\gamma \cdot \infty}^{\gamma \cdot \infty}\left|x^{k}\left(\partial^{e} g\right)(x)\right| d_{q} x \leq\left(\int_{-\gamma \cdot \infty}^{\gamma \cdot \infty}\left|x^{k} g(x)\right| d_{q} x+r^{k} B\right) \frac{2^{e}}{\gamma^{e}(1-q)^{e}}
$$

for every e and $k$ in $\mathbf{Z}_{\geq 0}$. Hence $\partial^{e} g \in \mathcal{H}_{a}^{\mathrm{D}} \mathcal{I}_{\gamma}^{\infty}$.

If $g \in \mathcal{H}_{a}^{\mathrm{D}} \mathcal{I}_{\gamma}$ but not necessarily in $\mathcal{I}_{\gamma}^{\infty}$, the above conclusion still holds for $k=0$ and $e \in \mathbf{Z}_{\geq 0}$. Then $\partial^{e} g \in \mathcal{H}_{a}^{\mathrm{D}} \mathcal{I}_{\gamma}$.

Proof. If $e=0$ the statement is trivial. Let $e>0$ and $g \in \mathcal{H}_{a}^{\mathrm{D}} \mathcal{I}_{\gamma}^{\infty}$. Then for every $r \in(\gamma, a)$ one has:

$$
\begin{aligned}
& \int_{-\gamma \cdot \infty}^{\gamma \cdot \infty}|x|^{k}\left|\left(\partial^{e} g\right)(x)\right| d_{q} x=\int_{-\gamma}^{\gamma}\left|x^{k}\left(\partial^{e} g\right)(x)\right| d_{q} x \\
& +(1-q) \sum_{s \leq-1} \sum_{\epsilon= \pm 1} q^{s(k+1)} \gamma^{k+1} \frac{\left|\left(\partial^{e-1} g\right)\left(\epsilon q^{s} \gamma\right)-\left(\partial^{e-1} g\right)\left(\epsilon q^{s+1} \gamma\right)\right|}{(1-q) q^{s} \gamma} \\
& \leq \sum_{s \leq-1} \sum_{\epsilon= \pm 1} q^{k s} \gamma^{k}\left[\left|\left(\partial^{e-1} g\right)\left(\epsilon q^{s} \gamma\right)\right|+\left|\left(\partial^{e-1} g\right)\left(\epsilon q^{s+1} \gamma\right)\right|\right]+\frac{2 r^{k-e} G(r) r \gamma}{(r-\gamma)(1-q)^{e}} \\
& \leq \frac{2}{\gamma(1-q)} \int_{-\gamma \cdot \infty}^{\gamma \cdot \infty}\left|x^{k}\left(\partial^{e-1} g\right)(x)\right| d_{q} x+\frac{2 r^{k} \gamma^{-e} G(r) r \gamma}{(r-\gamma)(1-q)^{e}}
\end{aligned}
$$

where the first inequality follows by estimate (3.1). Hence, by iterating the process we get:

$$
\begin{aligned}
& \int_{-\gamma \cdot \infty}^{\gamma \cdot \infty}\left|x^{k}\left(\partial^{e} g\right)(x)\right| d_{q} x \leq \frac{2^{2}}{\gamma^{2}(1-q)^{2}} \int_{-\gamma \cdot \infty}^{\gamma \cdot \infty}\left|x^{k}\left(\partial^{e-2} g\right)(x)\right| d_{q} x \\
& \quad+\frac{2 r^{k} \gamma^{-e} G(r) r \gamma}{(r-\gamma)(1-q)^{e}}+\frac{2^{2} r^{k} \gamma^{-e+1-1} G(r) r \gamma}{(r-\gamma)(1-q)^{e-1+1}} \leq \ldots \\
& \leq \frac{2^{e}}{\gamma^{e}(1-q)^{e}} \int_{-\gamma \cdot \infty}^{\gamma \cdot \infty}\left|x^{k}(g(x))\right| d_{q} x+\frac{2^{e} r^{k} \gamma^{-e} G(r) r \gamma}{(r-\gamma)(1-q)^{e}}\left[1+\frac{1}{2}+\cdots\left(\frac{1}{2}\right)^{e-1}\right] .
\end{aligned}
$$

Therefore we get the statement with $B=\frac{2 G(r) r \gamma}{(r-\gamma)}$. The conclusion for $k=0$ if $g \in \mathcal{H}_{a}^{\mathrm{D}} \mathcal{I}_{\gamma}$ is also clear from the proof. 
REMARK 3.6. If $g \in \mathcal{H}_{a}^{\mathrm{S}} \mathcal{I}_{\gamma}$, then $\int \partial^{k} g(x) \sim_{\gamma} 0$ and the $q$-integral of $g$ is always invariant under translation, in the notation of Section IV in [3]. In particular, this result will then follow without assuming condition (c) in that Section, since by the above Lemma the partial $q$-derivatives of $g(x)$ are automatically $q$-integrable.

From now on we will often use the shorthand notation $\int_{\gamma} f$ for $\int_{-\gamma \cdot \infty}^{\gamma \cdot \infty} f(t) d_{q} t$, see formula (1.1). We can conclude:

Proposition 3.7. Let $f \in \mathcal{I}_{\gamma^{\prime}}^{\omega}$ and $g \in \mathcal{H}_{a}^{\mathrm{D}} \mathcal{I}_{\gamma}$. Then $f *_{\gamma^{\prime}} g$ is well-defined and absolutely $q$-integrable on $L(\gamma)$, and $f *_{\gamma^{\prime}} g \in \mathcal{H}_{a}^{\mathrm{D}} \mathcal{I}_{\gamma}$.

Proof. Let $d:=2 /(\gamma(1-q))$. By Lemma 3.5 we know that $\int_{\gamma}\left|\partial^{e} g\right| \leq A d^{e}$ for some $A>0$. Hence for some $B>0$ we have that $\left|\left(\partial^{e} g\right)\left( \pm q^{-k} \gamma\right)\right| \leq B d^{e} q^{k}$ for every $e, k \in \mathbf{Z}_{\geq 0}$. Then the proof that $f *_{\gamma^{\prime}} g$ is well-defined on $L(\gamma)$ is similar to the second part of the proof of Lemma 3.4.

We still have to show that $\int_{\gamma}\left|f *_{\gamma^{\prime}} g\right|<\infty$. By assumption, $\left|\mu_{e, \gamma^{\prime}}(f)\right| \leq C q^{\frac{\alpha}{2} e^{2}} b^{e}$ for some constants $C, b$ and $\alpha>0$. Then

$$
\begin{aligned}
& \int_{\gamma}\left|\sum_{e=0}^{\infty} \frac{(-1)^{e} \mu_{e, \gamma^{\prime}}(f)}{[e]_{q} !} \partial^{e} g\right| \\
& \quad \leq \sum_{e=0}^{\infty} \frac{\left|\mu_{e, \gamma^{\prime}}(f)\right|}{[e]_{q} !} \int_{\gamma}\left|\partial^{e} g\right| \leq \frac{A C}{(q ; q)_{\infty}} \sum_{e=0}^{\infty} q^{\frac{\alpha}{2} e^{2}} d^{e} b^{e}(1-q)^{e}<\infty .
\end{aligned}
$$

Corollary 3.8. Let $f \in \mathcal{I}_{\gamma^{\prime}}^{\omega}$ and let $g \in \mathcal{H}_{a}^{\mathrm{D}} \mathcal{I}_{\gamma}$. Then $\partial^{k}\left(f *_{\gamma^{\prime}} g\right)$ and $f *_{\gamma^{\prime}} \partial^{k} g$ are also absolutely $q$-integrable on $L(\gamma)$ for every $k \in \mathbf{Z}_{\geq 0}$.

Proof. By Proposition 3.7 we know that $f *_{\gamma^{\prime}} g \in \mathcal{H}_{a}^{\mathrm{D}} \mathcal{I}_{\gamma}$. Hence $\partial^{k}\left(f *_{\gamma^{\prime}} g\right) \in \mathcal{H}_{a}^{\mathrm{D}} \mathcal{I}_{\gamma}$ by Lemma 3.5. By Lemma 3.5 we also know that $\partial^{k} g \in \mathcal{H}_{a}^{\mathrm{D}} \mathcal{I}_{\gamma}$, so that $f *_{\gamma^{\prime}} \partial^{k} g \in$ $\mathcal{H}_{a}^{\mathrm{D}} \mathcal{I}_{\gamma}$ by Proposition 3.7.

4. Associativity of $q$-convolution. The next step will be to investigate associativity. In order to do this, we need to know under which hypotheses $\left(f *_{\gamma} g\right) *_{\gamma} h$ and $f *_{\gamma}\left(g *_{\gamma} h\right)$ are well-defined. For $f *_{\gamma}\left(g *_{\gamma} h\right)$ this was essentially described in Lemma 3.4 and Proposition 3.7, i.e., $f$ and $g$ need to be of left type, and $h$ has to belong to $\mathcal{H}^{\mathrm{D}}$.

In order to understand when $\left(f *_{\gamma} g\right) *_{\gamma} h$ is well-defined, and to prove associativity, we need to investigate the behaviour of $\mu_{e, \gamma^{\prime}}\left(f *_{\gamma} g\right)$. We will use the following lemmas.

LEMMA 4.1. Let $f \in \mathcal{I}_{\gamma}^{\infty}$ such that $\partial^{j} f \in \mathcal{I}_{\gamma}^{\infty}$ for every $j \in \mathbf{Z}_{\geq 0}$. Then

$$
\int_{-\gamma \cdot \infty}^{\gamma \cdot \infty}\left(\partial^{a} f\right)(x) x^{b} d_{q} x= \begin{cases}(-1)^{a} q^{-b a+\frac{a^{2}-a}{2}} \frac{[b]_{q} !}{[b-a]_{q} !} \int_{-\gamma \cdot \infty}^{\gamma \cdot \infty} f(x) x^{b-a} d_{q} x & \text { if } b \geq a, \\ 0 & \text { otherwise. }\end{cases}
$$

In particular,

$$
\mu_{e+k, \gamma}\left(\partial^{k} f\right)=(-1)^{k} \frac{[e+k]_{q} !}{[e]_{q} !} \mu_{e, \gamma}(f) \quad \text { for every } k \in \mathbf{Z}_{\geq 0},
$$

and $\mu_{l, \gamma}\left(\partial^{k} f\right)=0$ if $l<k$. 
Proof. It is easy to see that $q^{b} X^{b} \partial f=\partial\left(f X^{b}\right)-f \partial\left(X^{b}\right)$ (this is a particular case of the braided Leibniz rule in [7] applied to $X^{b}$ and $f$ ). If we apply the unbounded $q$-integral to both sides with $f$ replaced by $\partial^{a-1} f$, then by absolute $q$-integrability we obtain:

$$
\int_{\gamma} X^{b} \partial^{a} f=-q^{-b}[b]_{q} \int_{\gamma} X^{b-1} \partial^{a-1} f .
$$

By repeating this procedure we get the statements of the Lemma.

LEMMA 4.2. If $f \in \mathcal{H}^{\mathrm{D}} \mathcal{I}_{\gamma, \alpha}^{\omega}$ then $X^{k} \partial^{j} f \in \mathcal{H}^{\mathrm{D}} \mathcal{I}_{\gamma, \alpha}^{\omega}$ for every $k \in \mathbf{Z}_{\geq 0}$.

Proof. By Lemma $3.5 X^{k} \partial^{j} f$ is absolutely $q$-integrable on $L(\gamma)$ for every $j$ and $k$. By Lemma $4.1 \mu_{k+e, \gamma}\left(\partial^{j} f\right)=0$ unless $e+k>j$. For $e+k>j$ we have

$$
\begin{aligned}
\left|\mu_{e, \gamma}\left(X^{k} \partial^{j} f\right)\right|= & \left|\mu_{k+e-j}(f)\right| q^{-\frac{k^{2}+2 e k+k}{2}} \frac{\left(q^{k+e-j+1} ; q\right)_{j}}{(1-q)^{j}} \\
& \leq\left[\frac{C q^{\frac{-k^{2}-k}{2}} b^{k-j}}{(1-q)^{j}} q^{\frac{\alpha}{2}(k-j)^{2}}\right] q^{-e(k-\alpha k+\alpha j)} q^{\frac{\alpha}{2} e^{2} b^{e}}
\end{aligned}
$$

for some $b>0$. Hence $\left|\mu_{e, \gamma}\left(X^{k} \partial^{j} f\right)\right|=O\left(q^{\frac{\alpha}{2} e^{2}}\left(b q^{-(k-\alpha k+\alpha j)}\right)^{e}\right)$ as $e \rightarrow \infty$.

It follows that $\partial^{k} f *_{\gamma} g$ is well-defined if $f \in \mathcal{H}_{a}^{\mathrm{D}} \mathcal{I}_{\gamma}^{\omega}$ and $g \in \mathcal{H}^{\mathrm{D}}$.

LEMMA 4.3. Let $f \in \mathcal{H}_{a}^{\mathrm{D}} \mathcal{I}_{\gamma}^{\omega}$ and let $g$ be defined, together with its $q$-derivatives, on a domain $\Omega$. Let $0 \neq x \in \Omega$ be such that, for some $R_{x}>0,\left|\partial^{e} g(x)\right|=O\left(R_{x}^{e}\right)$ as $e \rightarrow \infty$. Then, for every $k \in \mathbf{Z}_{\geq 0}, \partial^{k}\left(f *_{\gamma} g\right),\left(\partial^{k} f *_{\gamma} g\right)$ and $\left(f *_{\gamma} \partial^{k} g\right)$ are well defined at $x$ and

$$
\partial^{k}\left(f *_{\gamma} g\right)(x)=\left(\partial^{k} f *_{\gamma} g\right)(x)=\left(f *_{\gamma} \partial^{k} g\right)(x) .
$$

In particular, the result holds if $g \in \mathcal{H}^{\mathrm{D}} \mathcal{I}_{\gamma}$ and $x \in L(\gamma)$, or if $g$ is analytic on a starlike domain $\Omega$ centered at 0 and $x \in \Omega$.

Proof. Under the hypothesis on $f, g$ and $x$, the convolution product $f *_{\gamma} g$ is well defined at $x$ by the proof of Lemma 3.4 and Proposition 3.7. If $g$ and $x$ satisfy the hypothesis then also $\partial^{k} g$ and $x$ satisfy the hypothesis for every $k \in \mathbf{Z}_{\geq 0}$, hence $f *_{\gamma} \partial^{k} g$ and $\partial^{k}\left(f *_{\gamma} g\right)$ are well-defined at $x$. By Lemma $4.2\left(\partial^{k} f\right) *_{\gamma} g$ is also well defined at $x$. The three expressions are:

$$
\begin{gathered}
\left(\partial^{k}\left(f *_{\gamma} g\right)\right)(x)=\partial^{k}\left(\left(\sum_{e=0}^{\infty} \frac{(-1)^{e} \mu_{e, \gamma}(f)}{[e]_{q} !}\right) \partial^{e} g\right)(x)=\left(f *_{\gamma} \partial^{k} g\right)(x) \text { and } \\
\left(\partial^{k} f *_{\gamma} g\right)(x)=\sum_{e \geq 0} \frac{(-1)^{e+k} \mu_{e+k, \gamma}\left(\partial^{k} f\right)}{[e+k]_{q} !}\left(\partial^{e+k} g\right)(x) \\
=\sum_{e \geq 0} \frac{(-1)^{e} \mu_{e, \gamma}(f)}{[e]_{q} !}\left(\partial^{k+e} g\right)(x)=\left(f *_{\gamma} \partial^{k} g\right)(x)
\end{gathered}
$$

where absolute convergence follows by the conditions imposed on $x$ and $g$, hence the first statements.

If $g \in \mathcal{H}^{\mathrm{D}} \mathcal{I}_{\gamma}$ and $x \in L(\gamma)$ then it follows by the proof of Proposition 3.7 that $g$ and $x$ satisfy the conditions of the Lemma. 
If $g$ is analytic on a starlike domain $\Omega$ centered at 0 then the condition on $g$ and $x$ holds by the estimate in the proof of Lemma 3.4. Observe that in this case analytic continuation allows also $x=0 \in \Omega$.

Proposition 4.4. Let $f \in \mathcal{H}^{\mathrm{D}} \mathcal{I}_{\gamma^{\prime}}^{\omega}$ and $g \in \mathcal{H}_{a}^{\mathrm{D}} \mathcal{I}_{\gamma}^{\infty}$. Then $X^{k} \partial^{l}\left(f *_{\gamma^{\prime}} g\right) \in \mathcal{H}_{a}^{\mathrm{D}} \mathcal{I}_{\gamma}^{\infty}$ for all $k, l \in \mathbf{Z}_{\geq 0}$.

Proof. By Proposition $3.7 f *_{\gamma^{\prime}} g \in \mathcal{H}_{a}^{\mathrm{D}}$. Since $\partial^{l}\left(f *_{\gamma^{\prime}} g\right)=f *_{\gamma^{\prime}} \partial^{l} g$ by Lemma 4.3 and $\partial^{l} g \in \mathcal{H}_{a}^{\mathrm{D}} \mathcal{I}_{\gamma}^{\infty}$ by Lemma 3.5 , we might as well reduce to the case $l=0$. Then

$$
\begin{aligned}
& \int_{\gamma}\left|X^{k}\right|\left|f *_{\gamma^{\prime}} g\right|=\int_{\gamma}\left|X^{k}\right|\left|\sum_{e=0}^{\infty} \frac{(-1)^{e} \mu_{e, \gamma^{\prime}}(f)}{[e]_{q} !} \partial^{e} g\right| \\
& \leq C \int_{\gamma} \sum_{e=0}^{\infty}\left|X^{k}\right| \frac{q^{\frac{\alpha}{2}} e^{2} b^{e}}{[e]_{q} !}\left|\partial^{e} g\right|=C \sum_{e=0}^{\infty} \frac{q^{\frac{\alpha}{2} e^{2}} b^{e}}{[e]_{q} !} \int_{\gamma}\left|X^{k}\right|\left|\partial^{e} g\right|
\end{aligned}
$$

for some $C, \alpha, b>0$. Again by Lemma 3.5, there exists for any $r \in(\gamma, a)$ a constant $B>0$ such that

$$
\int_{\gamma}\left|X^{k}\right|\left|f *_{\gamma^{\prime}} g\right| \leq C \sum_{e=0}^{\infty} \frac{q^{\frac{\alpha}{2} e^{2}(2 b)^{e}}}{(q ; q)_{e} \gamma^{e}}\left(\int_{\gamma^{\prime}}\left|X^{k} f\right|+r^{k} B\right)<\infty .
$$

By the above results we know that for suitable $f$ and $g$ their convolution product $f *_{\gamma^{\prime}} g \in \mathcal{H}_{a}^{\mathrm{D}} \mathcal{I}_{\gamma}^{\infty}$. Next question is then whether $f *_{\gamma^{\prime}} g$ is of left type too. The answer is positive. We need the following Lemma.

Lemma 4.5. Let $f \in \mathcal{H}^{\mathrm{D}} \mathcal{I}_{\gamma}^{\omega}, g \in \mathcal{H}^{\mathrm{D}} \mathcal{I}_{\gamma^{\prime}}^{\omega}, k \in \mathbf{Z}_{\geq 0}$. Then

$$
\mu_{k, \gamma^{\prime}}\left(f *_{\gamma} g\right)=\sum_{e=0}^{k}\left[\begin{array}{l}
k \\
e
\end{array}\right]_{q} \mu_{e, \gamma}(f) \mu_{k-e, \gamma^{\prime}}(g) \text {. }
$$

Thus, if $\gamma=\gamma^{\prime}$ then $\int_{\gamma}\left(f *_{\gamma} g\right) X^{k}=\int_{\gamma}\left(g *_{\gamma} f\right) X^{k}$ for every $k \in \mathbf{Z}_{\geq 0}$ and $\int_{\gamma}\left(f *_{\gamma} g\right)=$ $\left(\int_{\gamma} f\right)\left(\int_{\gamma} g\right)$.

Proof. By Proposition $4.4\left(f *_{\gamma} g\right) X^{k}$ is absolutely $q$-integrable on $L\left(\gamma^{\prime}\right)$. Then

$$
\mu_{k, \gamma^{\prime}}\left(f *_{\gamma} g\right)=q^{\frac{k^{2}+k}{2}} \int_{\gamma^{\prime}}\left(f *_{\gamma} g\right) X^{k}=\int_{\gamma^{\prime}}\left(\sum_{e=0}^{\infty} \frac{(-1)^{e} \mu_{e, \gamma}(f)}{[e]_{q} !} X^{k} \partial^{e} g\right)
$$

Again by Lemma 3.5 and by dominated convergence we may interchange integration and summation over $e$. Then, by Lemma 4.1:

$$
\mu_{k, \gamma^{\prime}}\left(f *_{\gamma} g\right)=\sum_{e=0}^{k} \frac{\mu_{e, \gamma}(f)}{[e]_{q} !} \frac{q^{\frac{(k-e)^{2}+k-e}{2}}[k]_{q} !}{[k-e]_{q} !} \int_{\gamma^{\prime}} g X^{k-e}=\sum_{e=0}^{k}\left[\begin{array}{l}
k \\
e
\end{array}\right]_{q} \mu_{e, \gamma}(f) \mu_{k-e, \gamma^{\prime}}(g) .
$$

If $\gamma=\gamma^{\prime}$ then, by symmetry, the expression on the right equals $\mu_{k, \gamma}\left(g *_{\gamma} f\right)=$ $q^{\frac{k^{2}+k}{2}} \int_{\gamma}\left(g *_{\gamma} f\right) X^{k}$, hence the equality of the $q$-integrals. The last statement is formula (4.2) for $k=0$ and $\gamma=\gamma^{\prime}$.

Proposition 4.6. Let $f \in \mathcal{H}^{\mathrm{D}} \mathcal{I}_{\gamma, \alpha}^{\omega}$ and $g \in \mathcal{H}^{\mathrm{D}} \mathcal{I}_{\gamma^{\prime}, \beta}^{\omega}$. Then $f *_{\gamma} g \in \mathcal{H}^{\mathrm{D}} \mathcal{I}_{\gamma^{\prime}, \eta}^{\omega}$ with $\eta:=\frac{\alpha \beta}{\alpha+\beta}$. 
Proof. By Proposition 3.7, $f *_{\gamma} g \in \mathcal{H}_{a}^{\mathrm{D}} \mathcal{I}_{\gamma^{\prime}}$ for some $a>\gamma^{\prime}$ and by Proposition $4.4 f *_{\gamma} g \in \mathcal{I}_{\gamma^{\prime}}^{\infty}$. It follows by equation (4.2) that

$$
\left|\mu_{k, \gamma^{\prime}}\left(f *_{\gamma} g\right)\right| \leq \sum_{e=0}^{k}\left[\begin{array}{l}
k \\
e
\end{array}\right]_{q}\left|\mu_{e, \gamma}(f)\right|\left|\mu_{k-e, \gamma^{\prime}}(g)\right| .
$$

By using the fact that $\alpha e^{2}+\beta(k-e)^{2} \geq k^{2} \frac{\alpha \beta}{\alpha+\beta}$ we obtain that

$$
\left|\mu_{k, \gamma^{\prime}}\left(f *_{\gamma} g\right)\right| \leq C q^{k^{2} \frac{\alpha \beta}{2(\alpha+\beta)}} \sum_{e=0}^{k} b^{e} c^{k-e} \leq C q^{k^{2} \frac{\alpha \beta}{2(\alpha+\beta)}}(\max (b, c))^{k}(k+1)
$$

for some $b, c, C>0$.

REMARK 4.7. We have just seen that if $f \in \mathcal{H}^{\mathrm{D}} \mathcal{I}_{\gamma, \alpha}^{\omega}$ and $g \in \mathcal{H}^{\mathrm{D}} \mathcal{I}_{\gamma^{\prime}, \beta}^{\omega}$ then $f *_{\gamma} g \in \mathcal{H}^{\mathrm{D}} \mathcal{I}_{\gamma^{\prime}, \eta}^{\omega}$, where $\eta$ depends only on $\alpha$ and $\beta$ and is such that $\frac{1}{\eta}=\frac{1}{\alpha}+\frac{1}{\beta}$.

We are ready to show that our convolution is associative.

THEOREM 4.8. Let $f \in \mathcal{H}^{\mathrm{D}} \mathcal{I}_{\gamma}^{\omega}$ and $g \in \mathcal{H}^{\mathrm{D}} \mathcal{I}_{\gamma^{\prime}}^{\omega}$. Let $h$ be defined, together with its q-derivatives, on a domain $\Omega$ and let $x \in \Omega$ be such that, for some $R_{x}>0$, $\left|\left(\partial^{e} h\right)(x)\right|=O\left(R_{x}^{e}\right)$ as $e \rightarrow \infty$. Then $\left(\left(f *_{\gamma} g\right) *_{\gamma^{\prime}} h\right)(x)=\left(f *_{\gamma}\left(g *_{\gamma^{\prime}} h\right)\right)(x)$. In particular, the equality holds for every $x \in \Omega$ if $h$ is analytic on a starlike domain $\Omega$ centered at 0 and it holds for every $x \in L\left(\gamma^{\prime \prime}\right)$ if $h \in \mathcal{H}^{\mathrm{D}} \mathcal{I}_{\gamma^{\prime \prime}}^{\infty}$.

Proof. By the previous results all series involved converge absolutely for $x$ as in the hypothesis. We will show that the two given expressions coincide whenever they are well-defined. On the one hand

$$
\begin{aligned}
& \left(\left(f *_{\gamma} g\right) *_{\gamma^{\prime}} h\right)(x)=\sum_{l=0}^{\infty} \frac{(-1)^{l} \mu_{l, \gamma^{\prime}}\left(f *_{\gamma} g\right)}{[l]_{q} !}\left(\partial^{l} h\right)(x) \\
& =\sum_{l=0}^{\infty} \sum_{k=0}^{l} \frac{(-1)^{l} \mu_{k, \gamma}(f) \mu_{l-k, \gamma^{\prime}}(g)}{[k]_{q} ![l-k]_{q} !}\left(\partial^{e} h\right)(x)
\end{aligned}
$$

where we used equation (4.2) and Proposition 4.4.

On the other hand

$$
\begin{aligned}
\left(f *_{\gamma}\left(g *_{\gamma^{\prime}} h\right)\right)(x)=\sum_{l=0}^{\infty} \frac{(-1)^{l} \mu_{l, \gamma}(f)}{[l]_{q} !}\left(g *_{\gamma^{\prime}} \partial^{l} h\right)(x) \\
=\sum_{l=0}^{\infty} \sum_{m=0}^{\infty} \frac{(-1)^{l} \mu_{l, \gamma}(f)}{[l]_{q} !} \frac{(-1)^{m} \mu_{m, \gamma}(g)}{[m]_{q} !}\left(\partial^{l+m} h\right)(x) .
\end{aligned}
$$

The two expressions will coincide if for one of them the double sum is absolutely convergent. This is certainly the case for $x$ satisfying $\left|\partial^{e} h(x)\right|=O\left(R_{x}^{e}\right)$ as $e \rightarrow \infty$. This last condition is in particular satisfied if $h$ is analytic on a starlike domain $\Omega$ centered at 0 and $x \in \Omega$, or if $h \in \mathcal{H}^{\mathrm{D}} \mathcal{I}_{\gamma^{\prime \prime}}^{\infty}$ and $x \in L\left(\gamma^{\prime \prime}\right)$ (see also Lemma 4.3).

COROLlaRY 4.9. The class $\mathcal{H}^{\mathrm{D}} \mathcal{I}_{\gamma}^{\omega}$ is an algebra (not necessary unital) with respect to $*_{\gamma}$. Its subclass $\mathcal{H}^{\mathrm{S}} \mathcal{I}_{\gamma}^{\omega}$ is also an algebra (not necessary unital) and it is a left ideal of $\mathcal{H}^{\mathrm{D}} \mathcal{I}_{\gamma}^{\omega}$. 
Proof. By Theorem 4.8 the convolution product in $\mathcal{H}^{\mathrm{D}} \mathcal{I}_{\gamma}^{\omega}$ is associative. $\mathcal{H}^{\mathrm{S}} \mathcal{I}_{\gamma}^{\omega}$ is a subclass of functions of $\mathcal{H}^{\mathrm{D}} \mathcal{I}_{\gamma}^{\omega}$. By Lemma 3.4 and Proposition 4.6 the product of a function in $\mathcal{H}^{\mathrm{D}} \mathcal{I}_{\gamma}^{\omega}$ times a function in $\mathcal{H}^{\mathrm{S}} \mathcal{I}_{\gamma}^{\omega}$ is a function in $\mathcal{H}^{\mathrm{D}} \mathcal{I}_{\gamma}^{\omega}$ which is analytic on a whole strip around $\mathbf{R}$, hence it belongs to $\mathcal{H}^{\mathrm{S}} \mathcal{I}_{\gamma}^{\omega}$. By Theorem 4.8 and the uniqueness of analytic functions we have the statements.

We will see in Section 6, Example 6.7 that a left unit exists in $\mathcal{H}^{\mathrm{S}} \mathcal{I}_{\gamma}^{\omega}$.

5. Functions of strict left type. Recall Definition $3.1(\mathrm{~b})$ of the class $\mathcal{I}_{\gamma}^{\text {sw }}$ of functions of strict left type. These functions can be characterized in the following way.

Proposition 5.1. Let $f \in \mathcal{I}_{\gamma, \alpha}^{\mathrm{s} \omega}$. If $\alpha>1$ then $\left|f\left( \pm q^{-j} \gamma\right)\right|=0$ for every $j \in \mathbf{Z}$; if $\alpha=1$ then $\left|f\left( \pm q^{-j} \gamma\right)\right|=0$ for every $j \in \mathbf{Z}$ sufficiently large; if $0<\alpha<1$ then, for some $c>0,\left|f\left( \pm q^{-j} \gamma\right)\right|=O\left(q^{\frac{\beta}{2} j^{2}} c^{j}\right)$ as $j \rightarrow \infty$ with $\beta=\frac{1}{1-\alpha}$.

Conversely, let $f$ be a function on $L(\gamma)$ which is bounded on $\left\{ \pm q^{j} \gamma \mid j \geq 1\right\}$ and let $\beta>1$. If, for some $c>0,\left|f\left( \pm q^{-j} \gamma\right)\right|=O\left(q^{\frac{\beta}{2} j^{2} c^{j}}\right)$ as $j \rightarrow \infty$ then $f \in \mathcal{I}_{\gamma, \alpha}^{\text {s } \omega}$ with $\alpha=1-\beta^{-1}$.

Proof. Let $f \in \mathcal{I}_{\gamma, \alpha}^{\mathrm{s} \omega}$. Then there are constants $C, b>0$ such that

$$
q^{\frac{r^{2}+r}{2}} \sum_{k=-\infty}^{\infty} q^{k(1+r)} \gamma^{r+1}\left[\left|f\left(q^{k} \gamma\right)\right|+\left|f\left(-q^{k} \gamma\right)\right|\right] \leq C q^{\frac{\alpha}{2} r^{2}} b^{r} \quad \text { for every } r \in \mathbf{Z}_{\geq 0} .
$$

Hence every term of the sum on the left-hand side is dominated by the right-hand side. In particular,

$$
\left|f\left( \pm q^{-j} \gamma\right)\right| \leq C \gamma^{-r-1} q^{\frac{\alpha-1}{2} r^{2}+\frac{2 j-1}{2} r+j} b^{r}
$$

Therefore, if $\alpha>1$, or if $\alpha=1$ and $q^{j-\frac{1}{2}}<\gamma b^{-1}$, we have $\left|f\left( \pm q^{-j} \gamma\right)\right|=0$ since the left hand side does not depend on $r$. If $0<\alpha<1$, since the left hand side of (5.1) is independent of $r$, it will be in particular smaller than the right-hand side evaluated for $r:=\left[\frac{2 j-1}{2(1-\alpha)}\right] \geq 0$. Hence

$$
\begin{aligned}
& \left|f\left( \pm q^{-j} \gamma\right)\right| \leq C \gamma^{-1}\left(b \gamma^{-1}\right)^{r} q^{\frac{\alpha-1}{2} r^{2}+\frac{2 j-1}{2} r+j} \\
& \leq C^{\prime}\left(b \gamma^{-1}\right)^{\frac{j}{1-\alpha}} q^{-\frac{(2 j-1)^{2}(1-\alpha)}{8(1-\alpha)^{2}}+\frac{(2 j-1)^{2}}{4(1-\alpha)}+\frac{1}{2}} \\
& \leq C^{\prime} q^{\frac{1}{2}}\left(b \gamma^{-1}\right)^{\frac{j}{1-\alpha}} q^{\frac{(2 j-1)^{2}}{8(1-\alpha)}}=C^{\prime \prime}\left(\left(b \gamma^{-1} q^{-\frac{1}{2}}\right)^{\frac{1}{1-\alpha}}\right)^{j} q^{\frac{j^{2}}{2(1-\alpha)}}
\end{aligned}
$$

since $\frac{2 j-1}{2(1-\alpha)}-1 \leq r \leq \frac{2 j-1}{2(1-\alpha)}$, and the first statement follows.

Next we will prove the converse statement. By boundedness of $f$ on the set $\left\{ \pm q^{j} \gamma \mid\right.$ $j \geq 0\}$ it follows that, for some $M>0$, we have $\int_{-\gamma}^{\gamma}\left|t^{k} f(t)\right| d_{q} t \leq 2 M \gamma^{k+1}$ for all $k \in \mathbf{Z}_{\geq 0}$. Then,

$$
\begin{aligned}
& q^{\frac{k^{2}+k}{2}}\left(\int_{-\gamma \cdot \infty}^{\gamma \cdot \infty}-\int_{-\gamma}^{\gamma}\right)\left|x^{k} f(x)\right| d_{q} x=(1-q) q^{\frac{k^{2}+k}{2}} \sum_{j=1}^{\infty} \sum_{\epsilon= \pm 1} q^{-j(k+1)} \gamma^{k+1}\left|f\left(\epsilon q^{-j} \gamma\right)\right| \\
\leq & 2 C q^{\frac{k^{2}+k}{2}} \gamma^{k+1} \sum_{j=1}^{\infty} q^{j^{2} \frac{\beta}{2}-j(k+1)} c^{j} \\
= & 2 C q^{-\frac{1}{2 \beta}} \gamma^{k+1} q^{\frac{k}{2}-\frac{k}{\beta}} q^{\frac{k^{2}}{2}\left(1-\beta^{-1}\right)} \sum_{j=1}^{\infty} q^{\frac{\beta}{2}\left(j^{2}-2 j \frac{(k+1)}{\beta}+\frac{(k+1)^{2}}{\beta^{2}}\right)} c^{j} \leq M_{2} b^{k} q^{\frac{1}{2} \alpha k^{2}}
\end{aligned}
$$


for some $M_{2}, b>0$ and with $\alpha:=1-\beta^{-1}<1$, since the infinite sum is dominated by $s(\max (c, 1))^{s}+(\max (c, 1))^{s+1} \sum_{j=0}^{\infty} q^{\frac{j^{2}}{2(1-\alpha)}} c^{j}$, where $s:=\left[(k+1) \beta^{-1}\right] \geq 0$.

Remark 5.2. By Proposition 5.1 the pointwise product of functions $f \in \mathcal{I}_{\gamma, \alpha}^{\mathrm{s \omega}}$ and $g \in \mathcal{I}_{\gamma, \beta}^{\mathrm{s \omega}}$ satisfies the estimate $\left|(f g)\left( \pm q^{-j} \gamma\right)\right| \leq C c^{j} q^{\frac{j^{2}}{2}\left(\frac{1}{1-\alpha}+\frac{1}{1-\beta}\right)}$. Hence $f g \in$ $\mathcal{L}_{\gamma, \eta}^{\text {sw }}$, where $\eta=1$ if $\alpha$ or $\beta=1$, and $(1-\eta)^{-1}=(1-\alpha)^{-1}+(1-\beta)^{-1}>2$ if $\alpha, \beta<1$. Thus $\eta>\frac{1}{2}$ in all cases. This will be useful for finding examples in connection with commutativity of $q$-convolution (see Section 6).

EXAMPLE 5.3. As a consequence of Proposition 5.1 all functions belonging to $\mathcal{H}^{\mathrm{D}} \mathcal{I}_{\gamma, 1}^{\mathrm{s \omega}}$ must be of the form $f E_{q^{2}}\left(-q^{2 p} \gamma^{-2} X^{2}\right)$ for some $p \in \mathbf{Z}$ and some analytic function $f$.

REMARK 5.4. Observe that functions of strict left type $\alpha>1$ must be identically zero. From now on $\mathcal{H}^{\mathrm{D}} \mathcal{I}_{\gamma, \alpha}^{\mathrm{s} \omega}$ will automatically imply $\alpha \leq 1$.

EXAMPLE 5.5. The family of functions $f_{c}(c>0)$ given by $f_{c}(z):=e^{-c\left(\log \left(z^{2}+1\right)\right)^{2}}$ provides for every $\alpha \in(0,1)$ an example of a function which is of strict left type $\alpha$ on $L(\gamma)$ for each $\gamma>0$. Indeed, $f_{c} \in \mathcal{H}_{1}^{\mathrm{S}}$ and for every $\gamma>0$ there exists $b>0$ such that $\left|f_{c}\left( \pm q^{-k} \gamma\right)\right|=O\left(q^{\left(4 c \log \left(q^{-1}\right)\right) k^{2}} b^{k}\right)$ as $k \rightarrow \infty$. Hence $f \in \mathcal{H}_{1}^{\mathrm{S}} \mathcal{I}_{\gamma, \alpha}^{\mathrm{s} \omega}$ with $\alpha:=1-\left(4 c \log \left(q^{-1}\right)\right)^{-1} \in(0,1)$ if $c>\left(\left(4 \log \left(q^{-1}\right)\right)^{-1}\right.$.

EXAMPLE 5.6. Let $\mathcal{E}_{q^{2}}\left(-X^{2}\right)$ be the function defined by

$$
\mathcal{E}_{q^{2}}\left(-x^{2}\right):=\sum_{k=0}^{\infty} \frac{(-1)^{k} q^{\frac{k(k-1)}{2}} x^{2 k}}{\left(q^{2} ; q^{2}\right)_{k}}={ }_{1} \phi_{1}\left(0 ;-q ; q, x^{2}\right) .
$$

This function belongs with parameter value $1 / 2$ to a family of entire functions interpolating between $e_{q^{2}}\left(-X^{2}\right)$ and $E_{q^{2}}\left(-X^{2}\right)$, see also [1]. By formula (2.3) and Remark 2.4 in [12] with $z=-q$ and $n=2 k-1$ we have:

$$
(-q ; q)_{\infty}\left|\mathcal{E}_{q^{2}}\left(-q^{1-(2 k-1)}\right)\right| \leq q^{2 k^{2}-k}(-q ; q)_{\infty}^{2} .
$$

Hence, for $\gamma=1,\left|\mathcal{E}_{q^{2}}\left(-\left(q^{-k} \gamma\right)^{2}\right)\right|=O\left(q^{2 k^{2}}\right)$ as $k \rightarrow \infty$, which shows that $\mathcal{E}_{q^{2}}\left(-X^{2}\right)$ is of strict left type $3 / 4$ on $L(1)$.

LEMma 5.7. Let $f \in \mathcal{H}^{\mathrm{D}} \mathcal{I}_{\gamma, \alpha}^{\mathrm{s \omega}}$. Then, there are $C, c, R>0$ such that

$$
\nu_{e, \gamma}\left(\partial^{k} f\right) \leq C q^{\frac{\alpha}{2} e^{2}} c^{e} R^{k}
$$

for all $k, e \in \mathbf{Z}_{\geq 0}$. In particular, $\partial^{k} f \in \mathcal{H}^{\mathrm{D}} \mathcal{I}_{\gamma, \alpha}^{\text {sw }}$. that

Proof. Let $f \in \mathcal{H}_{a}^{\mathrm{D}} \mathcal{I}_{\gamma, \alpha}^{\mathrm{s} \omega}$ and $r \in(\gamma, a)$. By Lemma 3.5 there exists $B>0$ such

$$
q^{\frac{e^{2}+e}{2}} \int_{\gamma}\left|X^{e} \partial^{k} f\right| \leq q^{\frac{e^{2}+e}{2}} \frac{2^{k}}{\gamma^{k}(1-q)^{k}}\left[\int_{\gamma}\left|X^{e}\right||f|+r^{e} B\right]
$$


for all $k, e \in \mathbf{Z}_{\geq 0}$. Combination with Definition 3.1(b) immediately yields the result.

Proposition 5.8. Let $f \in \mathcal{H}^{\mathrm{D}} \mathcal{I}_{\gamma, \alpha}^{\omega}$ and $g \in \mathcal{H}^{\mathrm{D}} \mathcal{I}_{\gamma^{\prime}, \beta}^{\mathrm{s} \omega}$. Then $f *_{\gamma} g \in \mathcal{H}^{\mathrm{D}} \mathcal{I}_{\gamma^{\prime}, \beta}^{\mathrm{s} \omega}$.

Proof. For any $e \geq 0$,

$$
q^{\frac{e^{2}+e}{2}} \int_{\gamma^{\prime}}\left|f *_{\gamma} g\right|\left|X^{e}\right| \leq \sum_{k=0}^{\infty} \frac{\left|\mu_{k, \gamma}(f)\right|}{[k]_{q} !} q^{\frac{e^{2}+e}{2}} \int_{\gamma^{\prime}}|X|^{e}\left|\partial^{k} g\right| \leq C \sum_{k=0}^{\infty} \frac{q^{\frac{\alpha}{2} k^{2}} b^{k}}{[k]_{q} !} R^{k} q^{\frac{1}{2} \beta e^{2}} c^{e}
$$

by formula (1.3), Definition 3.1(b) and Lemma 5.7.

COROLlary 5.9. The class $\mathcal{H}^{\mathrm{D}} \mathcal{I}_{\gamma}^{\mathrm{s} \omega}$ is a subalgebra of $\mathcal{H}^{\mathrm{D}} \mathcal{I}_{\gamma}^{\omega}$. Its subclass $\mathcal{H}^{\mathrm{s}} \mathcal{I}_{\gamma}^{\mathrm{s} \omega}$ is a left ideal of $\mathcal{H}^{\mathrm{S}} \mathcal{I}_{\gamma}^{\omega}, \mathcal{H}^{\mathrm{D}} \mathcal{I}_{\gamma}^{\omega}$ and $\mathcal{H}^{\mathrm{D}} \mathcal{I}_{\gamma}^{\mathrm{s} \omega}$.

Proof. The proof is similar to that of Corollary 4.9

Definition 5.10. For every $c \in[0,1)$ we denote by $\mathcal{H}^{\mathrm{S}} \mathcal{I}_{\gamma,>c}^{\mathrm{s} \omega}$ the union of all spaces $\mathcal{H}^{\mathrm{S}} \mathcal{I}_{\gamma, \alpha}^{\mathrm{s} \omega}$ with $\alpha \in(c, 1]$.

Corollary 5.11. The classes $\mathcal{H}^{\mathrm{S}} \mathcal{I}_{\gamma,>c}^{\mathrm{s} \omega}($ for $c \in[0,1))$ and $\mathcal{H}^{\mathrm{S}} \mathcal{I}_{\gamma, c}^{\mathrm{s} \omega}($ for $c \in(0,1])$ are left ideals of $\mathcal{H}^{\mathrm{S}} \mathcal{I}_{\gamma}^{\mathrm{s} \omega}$ and of $\mathcal{H}^{\mathrm{S}} \mathcal{I}_{\gamma}^{\omega}$. Similar properties hold for $\mathcal{H}^{\mathrm{D}} \mathcal{I}_{\gamma}^{\mathrm{s} \omega}$.

Corollary 5.12. Let $f, g \in \mathcal{H}^{\mathrm{D}} \mathcal{I}_{\gamma}^{\omega}, h \in \mathcal{H}^{\mathrm{D}}$. Then $\left(f *_{\gamma} g *_{\gamma} h\right)(x)=\left(g *_{\gamma} f *_{\gamma}\right.$ $h)(x)$ for every $x$ where the product is defined. In particular, for every pair of ideals $I \subset J$ with $I, J \in\left\{\mathcal{H}^{\mathrm{S}} \mathcal{I}_{\gamma}^{\mathrm{s} \omega}, \mathcal{H}^{\mathrm{S}} \mathcal{I}_{\gamma}^{\omega}, \mathcal{H}^{\mathrm{D}} \mathcal{I}_{\gamma}^{\omega}, \mathcal{H}^{\mathrm{D}} \mathcal{I}_{\gamma}^{\mathrm{s} \omega}\right\}, I$ is a left module over $J /[J, J]_{*}$, where $[J, J]_{*}$ denotes the commutator ideal.

Proof. This is a consequence of Lemma 4.5 together with the fact that $f *_{\gamma} g$ depends only on the $q$-moments $\mu_{e, \gamma}(f)$ and not on the values of $f(x)$. The last statement follows by the inclusions of left ideals $\mathcal{H}^{\mathrm{S}} \mathcal{I}_{\gamma}^{\mathrm{s} \omega} \subset \mathcal{H}^{\mathrm{S}} \mathcal{I}_{\gamma}^{\omega} \subset \mathcal{H}^{\mathrm{D}} \mathcal{I}_{\gamma}^{\omega}$ and $\mathcal{H}^{\mathrm{S}} \mathcal{I}_{\gamma}^{\mathrm{s} \omega} \subset \mathcal{H}^{\mathrm{D}} \mathcal{I}_{\gamma}^{\mathrm{s} \omega} \subset \mathcal{H}^{\mathrm{D}} \mathcal{I}_{\gamma}^{\omega}$.

In particular, since $\mathcal{H}^{\mathrm{S}} \mathcal{I}_{\gamma,>c}^{\mathrm{s} \omega} \subset \mathcal{H}^{\mathrm{S}} \mathcal{I}_{\gamma,>d}^{\mathrm{s} \omega}$ (respectively $\mathcal{H}^{\mathrm{S}} \mathcal{I}_{\gamma, c}^{\mathrm{s} \omega} \subset \mathcal{H}^{\mathrm{s}} \mathcal{I}_{\gamma, d}^{\mathrm{s} \omega}$ ) for every $c \geq d$, we have a chain of left ideals on $\mathcal{H}^{\mathrm{s}} \mathcal{I}_{\gamma}^{\mathrm{s} \omega}=\mathcal{H}^{\mathrm{S}} \mathcal{I}_{\gamma,>0}^{\mathrm{s} \omega}$, and similarly for $\mathcal{H}^{\mathrm{D}} \mathcal{I}_{\gamma}^{\mathrm{s} \omega}$. Hence $E_{q^{2}}\left(-q^{2} X^{2}\right) \in \mathcal{H}^{\mathrm{S}} \mathcal{I}_{1,>c}^{\mathrm{s} \omega}$ for every $c<1$ and $e_{q^{2}}\left(-X^{2}\right) \in \mathcal{H}^{\mathrm{S}} \mathcal{I}_{\gamma,>\frac{1}{2}-\epsilon}^{\mathrm{s} \omega}$ for every $\gamma>0$ and $\epsilon \in(0,1 / 2)$.

6. Commutativity of $q$-convolution. We investigate commutativity now. We begin with a lemma.

LeMmA 6.1. Let $f \in \mathcal{H}^{\mathrm{D}} \mathcal{I}_{\gamma,>1 / 2}^{\mathrm{s} \omega}$ be such that $\int_{\gamma} f X^{k}=0$ for every $k \in \mathbf{Z}_{\geq 0}$. Then $f(x)=0$ for every $x$ in some neighbourhood of zero. In particular, if $f \in$ $\mathcal{H}^{\mathrm{S}} \mathcal{I}_{\gamma,>1 / 2}^{\mathrm{s} \omega}$, then $f(x)=0$ in each point $x$ of the strip around $\mathbf{R}$ where $f$ is analytic. Then

Proof. Let $f$ have power series $f(x)=\sum_{l} a_{l} x^{l}$ with radius of convergence $>\gamma$.

$$
\begin{aligned}
& \int_{\gamma}|f|^{2} E_{q^{2}}\left(-q^{2} X^{2} \gamma^{-2}\right)=\int_{-\gamma}^{\gamma}|f(x)|^{2} E_{q^{2}}\left(-q^{2} x^{2} \gamma^{-2}\right) d_{q} x \\
& =\int_{-\gamma}^{\gamma}\left(f(x) E_{q^{2}}\left(-q^{2} x^{2} \gamma^{-2}\right) \sum_{l=0}^{\infty} \bar{a}_{l} x^{l}\right) d_{q} x=\sum_{l=0}^{\infty} \bar{a}_{l} \int_{\gamma} f X^{l} E_{q^{2}}\left(-q^{2} \gamma^{-2} X^{2}\right) \\
& =\sum_{l=0}^{\infty} \bar{a}_{l} \sum_{p=0}^{\infty} \frac{(-1)^{p} q^{p^{2}+p} \gamma^{-2 p}}{\left(q^{2} ; q^{2}\right)_{p}} \int_{\gamma} f X^{2 p+l}=0 .
\end{aligned}
$$


Here the third and the fourth equality are justified by dominated convergence. Indeed,

$$
\sum_{l=0}^{\infty}\left|a_{l}\right| \int_{-\gamma}^{\gamma}|f(x)|\left|x^{l}\right| E_{q^{2}}\left(-q^{2} x^{2} \gamma^{-2}\right) d_{q} x \leq \sum_{l=0}^{\infty}\left|a_{l}\right| \gamma^{l} \int_{-\gamma}^{\gamma}|f(x)| d_{q} x<\infty
$$

and (use that $f$ has strict left type $>1 / 2$ )

$$
\sum_{p=0}^{\infty} \frac{q^{p^{2}+p} \gamma^{-2 p}}{\left(q^{2} ; q^{2}\right)_{p}} \int_{\gamma}\left|f X^{l+2 p}\right| \leq C \sum_{p=0}^{\infty} \frac{q^{p^{2}+p} \gamma^{-2 p}}{\left(q^{2} ; q^{2}\right)_{p}} q^{\left(\frac{1}{2} \alpha-\frac{1}{2}\right)(l+2 p)^{2}}\left(q^{-\frac{1}{2}} b\right)^{l+2 p}<\infty .
$$

Hence $|f(x)|^{2}=0$ if $x=\epsilon q^{k} \gamma$ with $k \in \mathbf{Z}_{\geq 0}$ and $\epsilon= \pm 1$. If moreover $f \in \mathcal{H}^{\mathrm{S}} \mathcal{I}_{\gamma,>1 / 2}^{\mathrm{s} \omega}$ then $f=0$ because it is analytic on a strip and vanishes on a sequence with limit point in the strip.

REMARK 6.2. Note the crucial role of analyticity of $f$ on a strip around $\mathbf{R}$ in order to conclude in the above Lemma that $f$ vanishes everywhere on $L(\gamma)$.

REMARK 6.3. The proof of the above Lemma showed that for any $f \in \mathcal{H}^{\mathrm{S}}$ the power series of $f E_{q^{2}}\left(-q^{2} \gamma^{-2} X^{2}\right)$ is absolutely $q$-integrable on $L(\gamma)$.

THEOREM 6.4. $\mathcal{H}^{\mathrm{S}} \mathcal{I}_{\gamma,>c}^{\mathrm{s} \omega}$ is a commutative algebra for every $c \in[1 / 2,1)$.

Proof. If $f, g \in \mathcal{H}^{\mathrm{S}} \mathcal{I}_{\gamma,>c}^{\mathrm{s} \omega}$ then by Lemma 4.5, Proposition 5.8 and Corollary 5.9 $f *_{\gamma} g-g *_{\gamma} f$ satisfies the hypothesis of Lemma 6.1.

The following Theorem shows that $\mathcal{H}_{1}^{\mathrm{S}} \mathcal{I}_{\gamma, 1 / 2}^{\mathrm{s} \omega}$ is far from commutative as a $q$ convolution algebra. Afterwards we give two other examples of noncommutativity. Recall that $Q$ is the $q$-shift $Q f(x)=f(q x)$.

Theorem 6.5. Let $g(x):=e_{q^{2}}\left(-x^{2}\right)$ (so $g \in \mathcal{H}_{1}^{\mathrm{S}} \mathcal{I}_{\gamma, 1 / 2}^{\mathrm{s} \omega}$ by Example 3.2 (a)). Let $f \in \mathcal{H}^{\mathrm{S}} \mathcal{I}_{\gamma,>1 / 2}^{\mathrm{s} \omega}$ be an entire function, not identically zero. Then $\left(Q^{-n} f\right) *_{\gamma} g \neq$ $g *_{\gamma}\left(Q^{-n} f\right)$ for $n$ sufficiently large.

Proof. For the discrete q-Hermite II polynomials

$$
\tilde{h}_{k}(x ; q):=x_{2}^{k} \phi_{1}\left(q^{-k}, q^{-k+1} ; 0 ; q^{2},-q^{2} x^{-2}\right)=(q ; q)_{k} \sum_{l=0}^{[k / 2]} \frac{(-1)^{l} q^{-2 l k+2 l^{2}+l} x^{k-2 l}}{\left(q^{2} ; q^{2}\right)_{l}(q ; q)_{k-2 l}}
$$

the following Rodrigues type formula was given in [11], formula (8.28):

$$
\left(\partial^{k} e_{q^{2}}\left(-X^{2}\right)\right)(x)=\frac{(-1)^{k} q^{\frac{k^{2}-k}{2}}}{(1-q)^{k}} \tilde{h}_{k}(x ; q) e_{q^{2}}\left(-x^{2}\right) .
$$

Also note by formula (II.6) in [6] that

$$
\tilde{h}_{k}(i ; q)=i_{2}^{k} \phi_{1}\left(q^{-k}, q^{-k+1} ; 0 ; q^{2}, q^{2}\right)=i^{k} q^{-\frac{1}{2} k(k-1)} .
$$

For $f \in \mathcal{I}_{\gamma}^{\omega}$ put

$$
\tilde{f}(x):=\sum_{k=0}^{\infty} \frac{q^{\frac{k^{2}-k}{2}} \mu_{k, \gamma}(f)}{(q ; q)_{k}} \tilde{h}_{k}(x ; q)
$$


It follows by the Rodrigues formula that

$$
\left(f *_{\gamma} e_{q^{2}}\left(-X^{2}\right)\right)(x)=\tilde{f}(x) e_{q^{2}}\left(-x^{2}\right)
$$

for $|\operatorname{Im}(x)|<1$. In fact, $\tilde{f}$ is an entire function, which we will show by uniform absolute convergence on compacta of the series defining $\tilde{f}(x)$. Indeed, for $k=2 h+\epsilon$ with $\epsilon=0,1$ :

$$
\begin{aligned}
& \tilde{h}_{k}(x ; q)=(q ; q)_{2 h+\epsilon} \sum_{l=0}^{h} \frac{(-1)^{l} q^{-2 l \epsilon-4 l h+2 l^{2}+l} x^{2 h+\epsilon-2 l}}{\left(q^{2} ; q^{2}\right)_{l}(q ; q)_{2 h+\epsilon-2 l}} \\
& =(-1)^{h}(q ; q)_{2 h+\epsilon} x^{\epsilon} q^{-2 h^{2}-2 h \epsilon+h} \sum_{p=0}^{h} \frac{(-1)^{p} q^{2 p^{2}-p+2 p \epsilon} x^{2 p}}{\left(q^{2} ; q^{2}\right)_{h-p}(q ; q)_{2 p}\left(1-q^{1+2 p}\right)^{\epsilon}} .
\end{aligned}
$$

Hence

$$
\begin{aligned}
\frac{q^{\frac{k^{2}-k}{2}}}{(q ; q)_{k}}\left|\tilde{h}_{k}(x ; q)\right| & \leq \frac{\left(q ; q^{2}\right)_{h}\left(q^{2} ; q^{2}\right)_{h}\left(1-q^{1+2 h}\right)^{\epsilon}|x|^{\epsilon}}{(q ; q)_{k}} \\
& \times \sum_{p=0}^{h} \frac{q^{2 p^{2}-p}|x|^{2 p}}{\left(q^{2} ; q^{2}\right)_{h}\left(q ; q^{2}\right)_{h}\left(q^{2} ; q^{2}\right)_{p}\left(1-q^{1+2 p}\right)^{\epsilon}} \leq \frac{\Phi(x)}{(q ; q)_{k}} \\
& \text { where } \Phi(x):=(1-q)^{-1} \max (1,|x|) \sum_{p=0}^{\infty} \frac{q^{2 p^{2}-p}|x|^{2 p}}{\left(q^{2} ; q^{2}\right)_{p}} .
\end{aligned}
$$

In combination with Definition 3.1(a) this shows that, for any $M>0$, the series $\sum_{k=0}^{\infty} \Phi(x) \frac{\left|\mu_{k, \gamma}(f)\right|}{(q ; q)_{k}}$ is uniformly convergent in $x$ for $|x| \leq M$.

Suppose that moreover $f \in \mathcal{I}_{\gamma}^{\mathrm{s} \omega}$. Substitute the $q$-integral for $\mu_{k, \gamma}(f)$ (see (1.2)) in equation (6.2). Then we can interchange $q$-integral and sum in the resulting expression, by combination of the above estimates with Definition 3.1(b). It follows that $\tilde{f}$ can be seen as a $q$-integral transform of $f$ with kernel $K(t, x)$ :

$$
\tilde{f}(x)=\int_{-\gamma . \infty}^{\gamma . \infty} f(t) K(t, x) d_{q} t, \quad \text { where } \quad \dot{K}(t, x):=\sum_{k=0}^{\infty} \frac{q^{k^{2}} t^{k} \tilde{h}_{k}(x ; q)}{(q ; q)_{k}} .
$$

By equations (6.1) and (2.4) we have $K(t, i)=E_{q}(i q t)$. Therefore

$$
\tilde{f}(i)=\int_{-\gamma \cdot \infty}^{\gamma \cdot \infty} f(t) E_{q}(i q t) d_{q} t .
$$

From now on assume that $f \in \mathcal{I}_{\gamma,>1 / 2}^{\mathrm{s} \omega}$ and entire, not identically zero. For $\lambda \neq 0$ put

$$
\tilde{f}_{\lambda}(x):=\lambda^{-1} \int_{-\gamma . \infty}^{\gamma . \infty} f\left(\lambda^{-1} t\right) K(t, x) d_{q} t=\int_{-\gamma \cdot \infty}^{\gamma . \infty} f(t) K(\lambda t, x) d_{q} t
$$

Then

$$
\tilde{f}_{\lambda}(i)=\int_{-\gamma \cdot \infty}^{\gamma \cdot \infty} f(t) E_{q}(i q \lambda t) d_{q} t=\sum_{k=0}^{\infty} q^{-\frac{k(k+1)}{2}} \mu_{k, \gamma}(f)(i \lambda)^{k}
$$


is well-defined for all $\lambda \in \mathbf{C}$ and entire in $\lambda$. If $\tilde{f}_{\lambda}(i)=0$ for $\lambda=q^{n_{k}}$, where $n_{k} \rightarrow \infty$ in $\mathbf{Z}$ as $k \rightarrow \infty$, then $\tilde{f}_{\lambda}(i)=0$ for all $\lambda \in \mathbf{C}$. Hence, by Lemma $6.1, f$ is identically zero, which contradicts our assumption. Thus for $n \in \mathbf{Z}$ sufficiently large we have $\tilde{f}_{q^{n}}(i) \neq 0$, hence $\left(Q^{-n} f\right) *_{\gamma} e_{q^{2}}\left(-X^{2}\right)=q^{n} e_{q^{2}}\left(-X^{2}\right) \tilde{f}_{q^{n}}$ does not extend to a function analytic at $i$. On the other hand $e_{q^{2}}\left(-X^{2}\right) *_{\gamma}\left(Q^{-n} f\right)$ is entire by Lemma 3.4. Hence the two products are different.

REMARK 6.6.

(a) Let $f \in \mathcal{I}_{\gamma}^{\text {s } \omega}$. Then we can express the kernel $K(t, x)$ (defined by (6.3)) also as a $q$-hypergeometric function:

$$
K(t, x)=(i q t ; q)_{\infty_{1}} \phi_{1}(i x, i q t ; q,-i q t x)=(i x,-i q t ; q)_{\infty_{2}} \phi_{1}\left(q t x^{-1}, 0 ;-i q t ; q, i x\right) .
$$

For the first identity use formula (3.29.12) in [9]. For the second identity use formula (III.1) in [6].

(b) Since there are functions $f$ and $g$ in $\mathcal{H}^{\mathrm{S}} \mathcal{I}_{\gamma, 1 / 2}^{\mathrm{s} \omega}$ for which $F:=f *_{\gamma} g-g *_{\gamma} f \not \equiv 0$, there exists a function $F \in \mathcal{H}^{\mathrm{S}} \mathcal{I}_{\gamma, 1 / 2}^{\text {s }}$, not identically zero, for which $\int_{\gamma} F X^{k}=0$ for every $k \in \mathbf{Z}_{\geq 0}$. In view of Lemma 6.1 we can state that, for $\alpha \in(0,1]$, the algebra $\mathcal{H}^{\mathrm{S}} \mathcal{I}_{\gamma, \alpha}^{\mathrm{s} \omega}$ is commutative iff each function $f$ in this algebra is determined by its moments $\mu_{e, \gamma}(f)\left(e \in \mathbf{Z}_{\geq 0}\right)$.

EXAMPLE 6.7. For $m \in \mathbf{Z}_{\geq 0}$ let

$$
\begin{gathered}
g_{m}(x):=e_{q^{2}}\left(-x^{2}\right)_{0} \phi_{1}\left(-; q^{1+2 m} ; q^{2},-q^{1+2 m} x^{2}\right) \\
=e_{q^{2}}\left(-x^{2}\right) \sum_{r=0}^{\infty} \frac{(-1)^{r} q^{2 r(r-1)} q^{(1+2 m) r} x^{2 r}}{\left(q^{1+2 m} ; q^{2}\right)_{r}\left(q^{2} ; q^{2}\right)_{r}} .
\end{gathered}
$$

Then $g_{m} \in \mathcal{H}_{1}^{\mathrm{S}}$. It will turn out that for each $\alpha>0$ the functions $g_{0}$ and $g_{1}$ are non-commuting elements of $\mathcal{H}_{1}^{\mathrm{S}} \mathcal{I}_{\gamma, \alpha}^{\omega}$. First we consider more generally $g_{m}$.

By the fact that $e_{q^{2}}\left(-X^{2}\right) \in \mathcal{I}_{\gamma, 1 / 2}^{\mathrm{s} \omega}$ we can estimate that, for some $b, C>0$,

$$
\begin{gathered}
\int_{\gamma}\left|g_{m} X^{k}\right| \leq \sum_{r=0}^{\infty} \frac{q^{2 r(r-1)} q^{(1+2 m) r}}{\left(q^{1+2 m} ; q^{2}\right)_{r}\left(q^{2} ; q^{2}\right)_{r}} q^{-\frac{1}{2}(2 r+k)(2 r+k+1)} \nu_{2 r+k, \gamma}\left(e_{q^{2}}\left(-X^{2}\right)\right) \\
\leq C q^{\frac{-k^{2}-k}{2}} b^{k} \sum_{r=0}^{\infty} \frac{q^{\frac{1}{4}(2 r+k)^{2}}\left(b q^{m-1-k}\right)^{2 r}}{\left(q^{1+2 m} ; q^{2}\right)_{r}\left(q^{2} ; q^{2}\right)_{r}}<\infty
\end{gathered}
$$

Hence $g_{m} \in \mathcal{I}_{\gamma}^{\infty}$ for every $\gamma>0$. By formula $(2.9), \mu_{2 k+1, \gamma}\left(g_{m}\right)=0$ for every $k \in \mathbf{Z}_{\geq 0}$. Furthermore, by dominated convergence we have

$$
\begin{aligned}
& \mu_{2 k, \gamma}\left(g_{m}\right)=q^{2 k^{2}+k} \sum_{r=0}^{\infty} \frac{(-1)^{r} q^{2 r(r-1)} q^{(1+2 m) r}}{\left(q^{1+2 m} ; q^{2}\right)_{r}\left(q^{2} ; q^{2}\right)_{r}} \int_{\gamma} X^{2 k+2 r} e_{q^{2}}\left(-X^{2}\right) \\
& =c_{q}(\gamma) q^{k^{2}+k}\left(q ; q^{2}\right)_{k} \sum_{r=0}^{\infty} \frac{\left(q^{1+2 k} ; q^{2}\right)_{r}(-1)^{r} q^{r^{2}-r} q^{(2 m-2 k) r}}{\left(q^{1+2 m} ; q^{2}\right)_{r}\left(q^{2} ; q^{2}\right)_{r}} \\
& =c_{q}(\gamma) q^{k^{2}+k}\left(q ; q^{2}\right)_{k 1} \phi_{1}\left(q^{1+2 k} ; q^{1+2 m} ; q^{2}, q^{2 m-2 k}\right) \\
& =c_{q}(\gamma) q^{k^{2}+k}\left(q ; q^{2}\right)_{k} \frac{\left(q^{2 m-2 k} ; q^{2}\right)_{\infty}}{\left(q^{1+2 m} ; q^{2}\right)_{\infty}}
\end{aligned}
$$


for every $k \in \mathbf{Z}_{\geq 0}$, where we used formula (2.9) and formula (II.5) in [6]. Hence

$$
\mu_{e, \gamma}\left(g_{m}\right)=\frac{c_{q}(\gamma)\left(q^{2} ; q^{2}\right)_{\infty}}{\left(q^{2 m+1} ; q^{2}\right)_{\infty}} \frac{q^{k^{2}+k}\left(q ; q^{2}\right)_{k}}{\left(q^{2} ; q^{2}\right)_{m-k-1}} \quad \text { if } e=2 k \text { with } k=0,1, \ldots, m-1,
$$

and $\mu_{e, \gamma}\left(g_{m}\right)=0$ otherwise. Hence $g_{m} \in \mathcal{I}_{\gamma, \alpha}^{\omega}$ for each $\alpha>0$ and

$$
\left(g_{m} *_{\gamma} f\right)(x)=\frac{c_{q}(\gamma)\left(q^{2} ; q^{2}\right)_{\infty}}{\left(q^{2 m+1} ; q^{2}\right)_{\infty}} \sum_{k=0}^{m-1} \frac{q^{k^{2}+k}}{\left(q^{2} ; q^{2}\right)_{k}\left(q^{2} ; q^{2}\right)_{m-k-1}}\left(\partial^{2 k} f\right)(x)
$$

for any function $f$ and for $x$ such that the $q$-derivatives of $f$ at $x$ are well-defined. In particular,

$$
g_{0} *_{\gamma} f=0, \quad \text { and } \quad g_{1} *_{\gamma} f=\frac{c_{q}(\gamma)\left(q^{2} ; q^{2}\right)_{\infty}}{\left(q^{3} ; q^{2}\right)_{\infty}} f \text { for all } f
$$

Hence, $g_{0} *_{\gamma} g_{1}=0 \neq g_{1} *_{\gamma} g_{0}$.

It follows from formulas (6.5) and (2.4) that

$$
g_{0}(x)=e_{q^{2}}\left(-x^{2}\right) \operatorname{Re}\left(E_{q}(i x)\right)=\operatorname{Re}\left(\frac{(-i x ; q)_{\infty}}{\left(-x^{2} ; q^{2}\right)_{\infty}}\right)=\operatorname{Re}\left(\frac{1}{(i x ; q)_{\infty}}\right)=\operatorname{Re}\left(e_{q}(i x)\right) .
$$

for $x \in \mathbf{R}$. Hence

$$
g_{0}\left( \pm \gamma q^{-k}\right)=\operatorname{Re}\left(\frac{1}{\left( \pm i \gamma q^{-k} ; q\right)_{\infty}}\right)=\operatorname{Re}\left(\frac{( \pm i)^{k} \gamma^{-k} q^{\frac{1}{2} k(k+1)}}{\left( \pm i \gamma^{-1} q ; q\right)_{k}( \pm i \gamma ; q)_{\infty}}\right) \quad \text { for } k \in \mathbf{Z}_{\geq 0}
$$

Therefore, the estimate $\left|g_{0}\left( \pm \gamma q^{-k}\right)\right|=O\left(q^{\frac{\beta}{2} k^{2}} c^{k}\right)$ as $k \rightarrow \infty$ is valid for some $c>0$ if and only if $\beta \leq 1$. This result combined with Proposition 5.1 implies that $g_{0}$ is not of strict left type on $L(\gamma)$.

The vanishing of all $q$-moments of $g_{0}$ can also be seen directly from the formula

$$
\int_{-\gamma \cdot \infty}^{\gamma \cdot \infty} x^{n} e_{q}( \pm i x) d_{q} x=\int_{-\gamma \cdot \infty}^{\gamma \cdot \infty} x^{n} e_{q^{2}}\left(-x^{2}\right) E_{q}( \pm i x) d_{q} x=0 \quad\left(n \in \mathbf{Z}_{\geq 0}, \gamma>0\right),
$$

which follows from formula (8.21) in [11] by substitution of $t= \pm q^{-1}$. Hence

$$
\int_{-\gamma \cdot \infty}^{\gamma \cdot \infty} x^{n} g_{0}(x) d_{q} x=\operatorname{Re} \int_{-\gamma \cdot \infty}^{\gamma \cdot \infty} x^{n} e_{q}(i x) d_{q} x=0
$$

EXAMPLE 6.8. Define a function $g$ on $L(1)$ by

$$
g\left( \pm q^{k}\right):=(-1)^{k} q^{k} e_{q^{2}}\left(-q^{2 k}\right) \quad(k \in \mathbf{Z})
$$

Then $g$ cannot be extended to a function in $\mathcal{H}^{\mathrm{D}}$ since it is alternating on a sequence approaching to zero. On the other hand,

$$
\left|g\left( \pm q^{-k}\right)\right|=\frac{q^{-k}}{\left(-q^{-2 k} ; q^{2}\right)_{\infty}}=\frac{q^{k^{2}}}{\left(-q^{2} ; q^{2}\right)_{k}\left(-1 ; q^{2}\right)_{\infty}}=O\left(q^{k^{2}}\right) \quad \text { as } k \rightarrow \infty
$$


and $\left|g\left( \pm q^{k}\right)\right| \leq 1$ if $k \in \mathbf{Z}_{\geq 0}$. Hence, by Proposition 5.1 it follows that $g \in \mathcal{I}_{1, \frac{1}{2}}^{\mathrm{s} \omega}$. Clearly, $\mu_{2 n+1,1}(g)=0$ for all $n \in \mathbf{Z}_{\geq 0}$. Furthermore,

$$
\begin{aligned}
\mu_{2 n, 1}(g) & =2(1-q) \sum_{k=-\infty}^{\infty}(-1)^{k} q^{(2 n+2) k} e_{q^{2}}\left(-q^{2 k}\right) \\
& =\frac{2(1-q)}{\left(-1 ; q^{2}\right)_{\infty}}{ }_{1} \psi_{1}\left(-1,0 ; q^{2},-q^{2 n+2}\right)=0
\end{aligned}
$$

for $n \in \mathbf{Z}_{\geq 0}$, where we used Ramanujan's ${ }_{1} \psi_{1}$ summation formula, see (II.29) in [6]. (C. Berg [2] used the same vanishing case of the ${ }_{1} \psi_{1}$ in connection with the indeterminate moment problem related to discrete $q$-Hermite II polynomials.) We conclude that $g *_{1} f=0$ for every $f$.

Next we consider $f *_{1} g$ for some $f \in \mathcal{I}_{1}^{\omega}$. Since $g$ is not in $\mathcal{H}^{\mathrm{D}}$, we cannot use the results of Section 3 in order to be sure that $\left(f *_{1} g\right)(x)$ is well-defined for suitable $x$. However, we can reason as follows. From the inequality $|(\partial h)(x)| \leq \frac{|h(x)|+|h(q x)|}{(1-q)|x|}$ and the definition of $g$ we see by induction with respect to $e$ that

$$
\left|\left(\partial^{e} g\right)\left( \pm q^{k}\right)\right| \leq e_{q^{2}}\left(-q^{2 k}\right) \frac{3^{e} q^{-k(e-1)}}{(1-q)^{e}} \quad\left(k, e \in \mathbf{Z}_{\geq 0}\right)
$$

Hence $\left(f *_{1} g\right)\left( \pm q^{k}\right)$ is well-defined for $f \in \mathcal{I}_{1}^{\omega}, k \in \mathbf{Z}_{\geq 0}$. Since $(-1)^{k} g\left(q^{k}\right)>0$ for all $k \in \mathbf{Z}$, one sees that $(-1)^{k}\left(\partial^{e} g\right)\left(q^{k}\right)>0$ for all $k, e \in \mathbf{Z}_{\geq 0}$. Hence $\left(f *_{1} g\right)\left(q^{2 k}\right)>0$ for $k \in \mathbf{Z}_{\geq 0}$ if $f \in \mathcal{I}_{1}^{\omega}$ is even and strictly positive (for instance $f:=e_{q^{2}}\left(-X^{2}\right)$ ). Again we have obtained a couterexample to commutativity of convolution.

Hence we have shown by means of Theorem 6.5 and Examples 6.7, 6.8 that none of the hypotheses of Theorem $6.4\left(f, g \in \mathcal{I}_{\gamma,>1 / 2}^{\mathrm{s} \omega} ; f \in \mathcal{I}_{\gamma}^{\mathrm{s} \omega} ; f \in \mathcal{H}^{\mathrm{D}}\right)$ can be relaxed.

7. $q$-Convolution and $q$-Fourier transform. In Remark 2.3 we introduced in (2.5) a $q$-Fourier transform pair with the second transform being given by

$$
\left(\mathcal{F}_{\gamma} f\right)(y):=\int_{-\gamma . \infty}^{\gamma . \infty} E_{q}(i q x y) f(x) d_{q} x .
$$

The right-hand side of (7.1) can be formally rewritten by power series expansion (2.4) of $E_{q}(i q x y)$ and by substitution of formula (1.2) for $\mu_{k, \gamma}(f)$. This defines the following transform:

$$
\left(\tilde{\mathcal{F}}_{\gamma} f\right)(y):=\sum_{k=0}^{\infty} \mu_{k, \gamma}(f) \frac{(i y)^{k}}{(q ; q)_{k}} .
$$

The transform $\tilde{\mathcal{F}}_{\gamma}$ is essentially the transform $F_{S}^{\prime \prime}(\mathrm{id}, \gamma)$ in [3] for $n=1$, with the difference that in [3] $x$ and $y$ do not commute.

Proposition 7.1.

(a) If $f \in \mathcal{I}_{\gamma}^{\omega}$ then $\tilde{\mathcal{F}}_{\gamma} f$ is well-defined and it is an entire analytic function.

(b) If moreover $f \in \mathcal{I}_{\gamma}^{\mathrm{s} \omega}$ then $\mathcal{F}_{\gamma} f$ is also well-defined and $\mathcal{F}_{\gamma} f=\tilde{\mathcal{F}}_{\gamma} f$.

(c) Let $f \in \mathcal{I}_{\gamma}^{\omega}$. Then $\tilde{\mathcal{F}}_{\gamma} f=0$ iff $\mu_{k, \gamma}(f)=0$ for all $k \in \mathbf{Z}_{\geq 0}$.

(d) Let $f \in \mathcal{H}^{\mathrm{S}} \mathcal{I}_{\gamma,>1 / 2}^{\mathrm{s} \omega}$. Then $\mathcal{F}_{\gamma} f=0$ iff $f=0$.

(e) Let $f \in \mathcal{H}^{\mathrm{D}} \mathcal{I}_{\gamma}^{\omega}, g \in \mathcal{H}^{\mathrm{D}} \mathcal{I}_{\gamma^{\prime}}^{\omega}$. Then $f *_{\gamma} g \in \mathcal{H}^{\mathrm{D}} \mathcal{I}_{\gamma^{\prime}}^{\omega}$ and $\tilde{\mathcal{F}}_{\gamma}\left(f *_{\gamma} g\right)=\left(\tilde{\mathcal{F}}_{\gamma} f\right)\left(\tilde{\mathcal{F}}_{\gamma^{\prime}} g\right)$.

Proof. (a) follows by formula (7.1) and Definition 3.1(a). 
In order to prove (b), first substitute formula (1.2) for $\mu_{k, \gamma}(f)$ in formula (7.2). Then justify interchange of summation and $q$-integration by dominated convergence by using the estimate for $\nu_{k, \gamma}(f)$ in Definition 3.1(b). Finally use the power series expansion (2.4) for $E_{q}(i q x y)$.

(c) is evident from (a) together with (7.2).

(d) follows from (c) and Lemma 6.1.

The first statement in (e) follows from Proposition 4.6. The second statement follows by taking formula (7.2) for $f *_{\gamma} g$ and then substituting formula (4.2). Rearrangement of the double summation is justified by dominated convergence (use Definition 3.1(a)).

8. Appendix. In our treatment of $f *_{\gamma} g$ in this paper we usually required $g$ to be analytic on a neigbourhood of 0 , while proofs of lemmas and propositions only used that, for some $R>0,\left|\left(\partial^{k} g\right)(x)\right|=O\left(R^{k}\right)$ as $k \rightarrow \infty$, uniformly for $x \rightarrow 0$ in $L(\gamma)$. In this Appendix we show that the analyticity requirement on $g$ is not an essential restriction. In connection with the $k^{\text {th }} q$-derivative at 0 occurring in the next Proposition, see also Koekoek and Koekoek [8].

Proposition 8.1. Let $g$ be a function defined on the half $q$-lattice $L^{\epsilon}(\gamma)=\left\{\epsilon q^{k} \gamma\right\}$ for some $\gamma>0$ and $\epsilon \in\{ \pm 1\}$. Suppose that there exist constants $C>0$ and $r>\gamma$ such that

$$
\left|\left(\partial^{k} g\right)\left(\epsilon q^{t} \gamma\right)\right| \leq \frac{C r}{\left(r-q^{t} \gamma\right)} \frac{1}{r^{k}(1-q)^{k}}
$$

for every $k \geq 0$ and for every $t \in \mathbf{Z}$ for which $q^{t} \gamma<r$. Then the limit $l_{p}:=$ $\lim _{k \rightarrow \infty}\left(\partial^{p} g\right)\left(\epsilon q^{k} \gamma\right)$ exists and is finite for every $p \in \mathbf{Z}_{\geq 0}$, and there exists a unique analytic function $\tilde{g}$ on $\{x \in \mathbf{C}|| x \mid<r\}$ such that $\tilde{g}=g$ on $\left\{\epsilon q^{k} \gamma \mid q^{k} \gamma<r\right\}$.

If $g$ is defined on the whole q-lattice $L(\gamma)$ and if the condition above is satisfied for every $\epsilon \in\{ \pm 1\}$ and if $l_{p}:=\lim _{k \rightarrow \infty}\left(\partial^{p} g\right)\left(\epsilon q^{k} \gamma\right)$ is independent of $\epsilon$, then there exists a unique analytic function $\tilde{g}$ on $\{x \in \mathbf{C}|| x \mid<r\}$ such that $\tilde{g}=g$ on $\left\{ \pm q^{k} \gamma \mid q^{k} \gamma<r\right\}$. In particular, if $g$ is a function defined on $\mathbf{R}$ such that on every $q$-lattice $L(\gamma)$ the above conditions are satisfied and $l_{p}$ is independent of $\gamma$ for every $p \in \mathbf{Z}_{\geq 0}$ then $g$ is analytic.

Proof. It follows from the identity

$$
g\left(\epsilon q^{k} \gamma\right)=g\left(\epsilon q^{k+1} \gamma\right)+(1-q) \epsilon q^{k} \gamma(\partial g)\left(\epsilon q^{k} \gamma\right)
$$

that

$$
\left(\partial^{b} g\right)\left(\epsilon q^{k+p} \gamma\right)=\left(\partial^{b} g\right)\left(\epsilon q^{k} \gamma\right)-(1-q) \epsilon q^{k} \gamma \sum_{m=0}^{p-1} q^{m}\left(\partial^{b+1} g\right)\left(\epsilon q^{k+m} \gamma\right)
$$

for every $b, p \in \mathbf{Z}_{\geq 0}$ and $k \in \mathbf{Z}$. For $k$ such that $q^{k} \gamma<r$ it follows by (8.1) that $\sum_{m=0}^{\infty} q^{m}\left(\partial^{b+1} g\right)\left(\epsilon q^{k+m} \gamma\right)$ is absolutely convergent. Hence both sides of equation (8.2) converge to a finite limit $l_{b}$ as $p \rightarrow \infty$.

It follows by induction with respect to $n$ that

$$
g(x)=\sum_{k=0}^{n}\left[\begin{array}{l}
n \\
k
\end{array}\right]_{q}(1-q)^{k} x^{k}\left(\partial^{k} g\right)\left(q^{n-k} x\right)
$$


Let $x \in L(\gamma),|x|<r$. Then

$$
\begin{gathered}
{\left[\begin{array}{l}
n \\
k
\end{array}\right]_{q}(1-q)^{k}\left|x^{k}\left(\partial^{k} g\right)\left(q^{n-k} x\right)\right| \leq \frac{(1-q)^{k}}{(q ; q)_{k}}|x|^{k} \frac{C r}{r-q^{n-k}|x|} \frac{1}{r^{k}(1-q)^{k}}} \\
\leq \frac{C r}{r-|x|} \frac{1}{(q ; q)_{k}}\left(\frac{|x|}{r}\right)^{k} .
\end{gathered}
$$

Hence by dominated convergence we can take the limit for $n \rightarrow \infty$ and $g(x)=$ $\sum_{k=0}^{\infty} \frac{x^{k} l_{k}}{[k]_{q} !}$ for $x=\epsilon q^{k} \gamma$ with $q^{k} \gamma<r$.

The other statements follow from the definition of $\tilde{g}(x)=\sum_{k=0}^{\infty} \frac{x^{k} l_{k}}{[k]_{q} !}$.

EXAMPLE 8.2. Let $c_{q, \gamma}(x)$ be defined on $L(\gamma)$ as:

$$
c_{q, \gamma}\left(\epsilon q^{k} \gamma\right):= \begin{cases}\int_{q^{k} \gamma} e_{q^{2}}\left(-X^{2}\right) & \text { if } \epsilon=1, \\ \int_{-q^{k} \gamma} e_{q^{2}}\left(-X^{2}\right) & \text { if } \epsilon=-1\end{cases}
$$

Then, since the $q$-integral is $q$-periodic, the limit for $k \rightarrow \infty$ of $c_{q, \gamma}\left( \pm q^{k} \gamma\right)=c_{q}(\gamma)$, and $\left(\partial^{p} c_{q, \gamma}\right)(x) \equiv 0$ on $L(\gamma)$. The power series $\tilde{c}_{q}(x)$ is then trivially the constant $c_{q}(\gamma)$ defined by (2.7). However, the function $c_{q}(z):=\int_{z} e_{q^{2}}\left(-X^{2}\right)$, coinciding with $c_{q, \gamma}$ on every $L(\gamma)$ for $\gamma>0$, is not analytic. Indeed the limit of $c_{q}(x)$ for $x \rightarrow 0$ cannot exist.

EXAmple 8.3. Consider the function $f\left(\epsilon q^{k} \gamma\right):=\left(-\epsilon q^{k} \gamma ; q\right)_{\infty}$ on $L(\gamma)$. Clearly $\lim _{k \rightarrow \infty} f\left(\epsilon q^{k} \gamma\right)=1$. One checks that $\left(\partial^{n} f\right)\left(\epsilon q^{k} \gamma\right)=\frac{q^{\frac{n(n-1)}{2}}}{(1-q)^{n}}\left(-\epsilon q^{k+n} \gamma ; q\right)_{\infty}$ so that the limit for $k \rightarrow \infty$ is well-defined. The majorization for $\left|\left(\partial^{n} f\right)\left(\epsilon q^{k} \gamma\right)\right|$ is clearly verified for every $n \in \mathbf{Z}_{\geq 0}, k \in \mathbf{Z}$ and $\gamma>0$. Hence $f$ can be extended to the power series $E_{q}(x)=\sum_{k=0}^{\infty} \frac{q^{\frac{k(k-1)}{2}} x^{k}}{(q ; q)_{k}}$. Since the limits for $k \rightarrow \infty$ of the $q$-derivatives do not depend on $\gamma$, we have checked that $E_{q}(x)=(-x ; q)_{\infty}$.

\section{REFERENCES}

[1] N. M. AtAKISHIYeV, On a one-parameter family of q-exponential functions, J. Phys. A: Math. Gen., 29 (1996), pp. L223-L227.

[2] C. BERG, On some indeterminate moment problems for measures on a geometric progression, J. Comput. Appl. Math., 99 (1998), pp. 67-75.

[3] G. CaRnovale, On the braided Fourier transform in the n-dimensional quantum space, J. Math. Phys., 40 (1999), pp. 5972-5997.

[4] G. Carnovale, Algebraic and Analytic Aspects of the Quantum Yang-Baxter Equation, Dissertation, University of Utrecht, 1999.

[5] G. Carnovale, On the q-convolution on the line, preprint, math.CA/9912113, 1999.

[6] G. Gasper and M. Rahman, Basic Hypergeometric Series, Cambridge University Press, Cambridge, 1990.

[7] A. KempF AND S. MAJID, Algebraic q-integration and Fourier theory on quantum and braided spaces, J. Math. Phys., 35 (1994), pp. 6802-6837.

[8] J. Ковковк And R. Kовковк, A note on the q-derivative operator, J. Math. Anal. Appl., 176 (1993), pp. 627-634.

[9] R. KoEkoEK AND R. F. SWARTtouw, The Askey-scheme of hypergeometric orthogonal polynomials and its q-analogue, Report 98-17, Faculty of Technical Mathematics and Informatics, Delft University of Technology, 1998.

[10] T. H. KooRNWINDER, Orthogonal polynomials in connection with quantum groups, in Orthogonal Polynomials: Theory and Practice, P. Nevai, ed., NATO ASI Series C 294, Kluwer Academic Press, 1990, pp. 257-292. 
[11] T. H. Koornwinder, Special functions and q-commuting variables. in Special Functions, $q$ Series and Related Topics, M. E. H Ismail, D. R. Masson, and M. Rahman, eds., Fields Institute Communications 14, American Mathematical Society, 1997, pp. 131-166.

[12] T. H. Koornwinder AND R. F. SwartTouw, On q-analogues of the Fourier and Hankel transforms, Trans. Amer. Math. Soc., 333 (1992), pp. 445-461.

[13] S. MAJID, Braided momentum in the q-Poincaré group, J. Math. Phys., 34 (1993), pp. 20452058.

[14] S. MAJID, Foundations of Quantum Groups, Cambridge University Press, 1995. 